\title{
Computationally Efficient Angle and Polarization Estimation in the Presence of Multipath Propagation Using Dual-Polarization Vector Sensor Array
}

\author{
Weijian Si, Pinjiao Zhao, Zhiyu Qu, and Liwei Wang \\ Department of Information and Communication Engineering, Harbin Engineering University, Harbin 150001, China \\ Correspondence should be addressed to Zhiyu Qu; quzhiyu@hrbeu.edu.cn
}

Received 30 December 2015; Revised 1 May 2016; Accepted 9 May 2016

Academic Editor: Shih Yuan Chen

Copyright (c) 2016 Weijian Si et al. This is an open access article distributed under the Creative Commons Attribution License, which permits unrestricted use, distribution, and reproduction in any medium, provided the original work is properly cited.

\begin{abstract}
This paper presents a computationally efficient angle and polarization estimation method for a mixture of uncorrelated and coherent sources using a dual-polarization vector sensor array. The uncorrelated sources are separated from the coherent sources on the basis of the modulus property of eigenvalues. The angles of the uncorrelated sources are estimated by employing rotational invariance and the associated polarization is obtained from the estimate of the uncorrelated array response matrix through elementwise division. For the distinguished coherent sources, two Hankel matrices are constructed from the elements of the estimated coherent array response matrix of each coherent group, from which two rotational-invariant submatrix pairs are extracted for estimating the coherent angles with a high precision. The least-square solution to the coherent polarization equation is derived for estimating the coherent polarization parameters. For each uncorrelated source and coherent group, the proposed method estimates the associated angle and polarization parameters separately, which avoids the need of 3D spectral search. In comparison with the existing methods, the simulation results show that the proposed method yields favorable performance in terms of computational efficiency and estimation accuracy.
\end{abstract}

\section{Introduction}

Direction of arrival (DOA) estimation has been a key issue in many practical applications such as radar, wireless communication system, and seismology $[1,2]$. The vector sensor array, which offers advantages such as good robustness towards channel fading and strong immunity to interference, has been introduced for DOA estimation [3-6]. Typically, the subspace-based methods such as multiple signal classification (MUSIC) [7] and estimation of signal parameters via rotation invariance techniques (ESPRIT) [8] can be extended for the vector sensor array. More specifically, the polarized MUSICbased methods [9-12] and the polarized ESPRIT-based methods [13-16] were demonstrated to have enhanced estimation performance as compared to the traditional MUSIC and ESPRIT methods with scalar sensor arrays. However, all the aforementioned methods operate under the assumption that the impinging sources are uncorrelated, while this assumption is often not true in practical scenarios due to multipath propagation. In such scenarios, the sources from the identical target may undergo reflection from various surfaces, and then the received sources may be a mixture of uncorrelated and coherent sources. Therefore, the performance of these methods would critically degrade because the existence of coherent sources directly causes the rank deficiency of the array covariance matrix.

To solve the aforementioned problem, several "decorrelation" methods have been reported in [17-21]. A polarization smoothing (PS) technique was first developed in [17] to handle the coherent sources by using a vector sensor array. Subsequently, a modified polarization difference smoothing (PDS) method [18] was proposed in conjunction with the propagator method. Theoretically, the maximum number of resolvable coherent sources of the PDS method was limited to six. $\mathrm{Gu}$ et al. [19] introduced a propagator-based angle and polarization estimation method with an extra isolated vector sensor. In practice, a major limitation of these methods is their focus on addressing the coherent sources, while the coexistence of both uncorrelated and coherent sources is a common 
scenario for many practical applications owing to multipath propagation [22]. Another method for coherent sources, called polarization angular smoothing algorithm (PAS) [20], utilizes the cross-correlations among six subarrays and can be extended for scenarios of coexistent uncorrelated and coherent sources. Unfortunately, the simultaneous estimation of the uncorrelated and coherent sources using this method results in mutual interference between uncorrelated and coherent sources and poor utilization of the array aperture. Following the technique of [20], an improved polarization angular smoothing algorithm (IPAS) [21] was developed with an extended array aperture, which takes special consideration of the coexistence of uncorrelated and coherent sources. However, a loss of power may occur at the coherent sources due to the utilization of spatial differencing technique. All of these aforementioned "decorrelation" methods operate with spatially collocated six-component vector sensor arrays that are easily subjected to mutual coupling across the collocated antennas, and the utilization of the six-component vector sensor array results in an increase in hardware costs for antenna arrays. Moreover, these methods are unable to estimate the polarization parameters that are useful for angular resolution, target classification, and recognition [23].

To address these issues, we present a novel method in this paper for jointly estimating the angle and the polarization under the coexistence of the uncorrelated and coherent sources in this paper using a dual-polarization uniform linear array (ULA). On the basis of the modulus property of eigenvalues and rotational invariance, the uncorrelated sources are firstly distinguished from the coherent sources, and their corresponding angles are estimated. Subsequently, the uncorrelated array response matrix corresponding to each uncorrelated source is obtained from the estimated source subspace and the associated eigenvector matrix. The associated polarization parameters are obtained by exploiting the inherent relationship between the odd and even rows of the uncorrelated array response matrix using elementwise division operation. For the remaining coherent sources, the array response matrix of each coherent group is estimated in a way similar to that followed for the uncorrelated array response matrix, with which two Hankel matrices are constructed for "decorrelation." The angles of each coherent group are estimated via construction of two pairs of rotational-invariant submatrices. Finally, the least-square solution to the coherent polarization equation is derived, and the associated coherent polarization parameters are obtained accordingly. To be more specific, the main contributions of this paper are as follows:

(1) The proposed method considers the joint angle and polarization estimation for a mixture of uncorrelated and coherent sources, unlike most of the existing "decorrelation" methods that focus only on the angle estimation of coherent sources.

(2) By utilizing a dual-polarization vector sensor array, the problem of mutual coupling across the collocated antennas is alleviated. In addition, the costs of antenna hardware are also reduced.

(3) By employing the modulus property of eigenvalues, the uncorrelated sources are distinguished from the

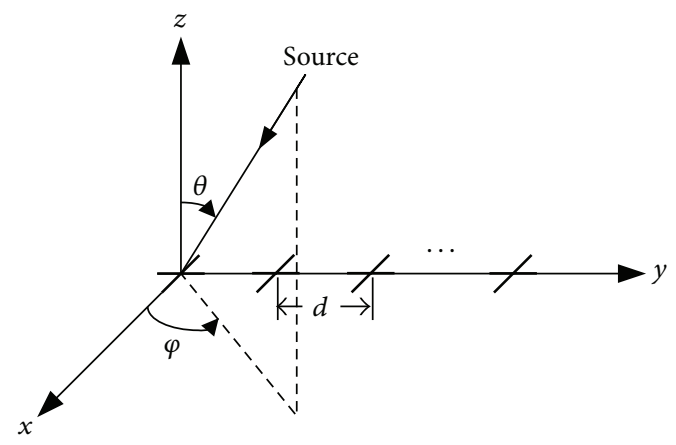

FIGURE 1: Uniform linear array of dual-polarization vector sensor.

coherent sources without a loss of power at coherent sources. Based on estimated array response matrix of each uncorrelated source and that of each coherent group, the corresponding angle and polarization parameters are estimated separately, which avoids the need of three-dimensional (3D) spectral search.

(4) The identifiability and computational complexity of the proposed method are discussed.

The mathematical notations used throughout this paper are as follows. Vectors and matrices are, respectively, denoted by lowercase and uppercase bold-faced italicized letters. $(\cdot)^{T}$, $(\cdot)^{*},(\cdot)^{H},(\cdot)^{-1},(\cdot)^{\dagger}, \otimes$, and $E\{\cdot\}$ represent transpose, conjugate, conjugate transpose, inverse, Moore-Penrose inverse, Kronecker product, and the statistical expectation, respectively. $\mathbf{0}_{m \times n}$ represents $m \times n$ null matrix, $\mathbf{I}_{m} m \times m$ identity matrix, $\|\cdot\|_{F}$ the Frobenius norm, and ./ the elementwise division. Besides, $\partial f(\mathbf{F}) / \partial \mathbf{F}$ denotes the derivative of $f(\mathbf{F})$ with respect to the matrix $\mathbf{F}$; $\operatorname{diag}\{\cdot\}$ and blkdiag $\{\cdot\}$ denote a diagonal matrix and a block diagonal matrix, respectively. $\mathbf{F}(a: b, c$ : $d$ ) denotes a submatrix constructed by the elements from rows $a$ to $b$ and columns $c$ to $d$ of $\mathbf{F}$.

The rest of the paper is organized as follows. The angle and polarization estimation model in the presence of multipath propagation is formulated in Section 2. Section 3 presents the details of the proposed angle and polarization estimation method under the coexistence of uncorrelated and coherent sources. The identifiability and computational complexity of the proposed method are discussed in Section 4. Section 5 discusses the simulation performance of the proposed method. Conclusions are drawn in Section 6.

\section{Problem Formulation}

Consider a ULA consisting of $M$ dual-polarization sensors (i.e., crossed dipoles) placed along the $y$-axis with interelement spacing $d$, as depicted in Figure 1. $\theta \in[0, \pi)$ and $\varphi \in[0,2 \pi)$ signify the elevation angle and the azimuth angle measured from the positive $z$ - and $x$-axes, respectively. For simplicity, it is assumed that the impinging source is in the $y-z$ plane; that is, $\varphi=0$. Assume that a total of $K$ completely polarized narrowband transverse electromagnetic (TEM) waves impinge on the ULA, and the electric field is described in Cartesian coordinate as

$$
\mathbf{E}=-E_{\varphi} \mathbf{e}_{x}+E_{\theta} \cos \theta \mathbf{e}_{y}-E_{\theta} \sin \theta \mathbf{e}_{z},
$$


where $\mathbf{e}_{x}, \mathbf{e}_{y}$, and $\mathbf{e}_{z}$ are the unit vectors along the $x$-, $y$-, and $z$-axes; $E_{\varphi}=E_{o} \cos \gamma$ and $E_{\theta}=E_{o} \sin \gamma e^{j \eta}$ are, respectively, the horizontal and the vertical components of a polarization ellipse. $E_{o}$ represents the source amplitude (an arbitrary nonzero complex constant) and $\gamma \in[0, \pi / 2)$ refers to the auxiliary polarization angle and $\eta \in[-\pi, \pi)$ refers to the polarization phase difference. The dual-polarization ULA configured in Figure 1 is known to only receive the electric field components of the waves along the $x$ - and $y$-axes. Therefore, the source vectors collected by the $m$ th vector sensor for polarization $x$ and polarization $y$ are, respectively, expressed as

$$
\begin{aligned}
x_{m}^{[x]}(t)= & -\sum_{k=1}^{K} s_{k}(t) \cos \gamma_{k} e^{-j 2 \pi(m-1) d \sin \theta_{k} / \lambda}+n_{m}^{[x]}(t), \\
x_{m}^{[y]}(t)= & -\sum_{k=1}^{K} s_{k}(t) \cos \theta_{k} \sin \gamma_{k} e^{j \eta_{k}} e^{-j 2 \pi(m-1) d \sin \theta_{k} / \lambda} \\
& +n_{m}^{[y]}(t),
\end{aligned}
$$

where $m \in[1, M], n_{m}^{[x]}(t)$, and $n_{m}^{[y]}(t)$ denote the noise components of polarization $x$ and polarization $y$, respectively, and $\lambda$ is the carrier wavelength. Combining (2) and (3), we have

$$
\mathbf{x}_{m}(t)=\left[\left(x_{m}^{[x]}(t)\right)^{T},\left(x_{m}^{[y]}(t)\right)^{T}\right]^{T}
$$

with $\mathbf{n}_{m}(t)=\left[\left(n_{m}^{[x]}(t)\right)^{T},\left(n_{m}^{[y]}(t)\right)^{T}\right]^{T}$ being the received noise vector. In realistic cases, the coherent sources are often susceptible to multipath propagation. The $K$ impinging sources, parameterized by $\left\{\theta_{1}, \gamma_{1}, \eta_{1}\right\},\left\{\theta_{2}, \gamma_{2}, \eta_{2}\right\}, \ldots,\left\{\theta_{K}, \gamma_{K}, \eta_{K}\right\}$, are composed of $D$ groups (each group having $p_{k}$ coherent sources) of $K_{c}$ coherent and $K_{u}$ uncorrelated sources, where $K_{c}=K-K_{u}=\sum_{k=1}^{D} p_{k}$. The entire $2 M \times 1$ array output vector of the ULA at time $t$ is written as

$$
\begin{aligned}
\mathbf{X}(t)= & \sum_{k=1}^{K_{u}} \mathbf{a}\left(\theta_{k}\right) s_{k}(t)+\sum_{k=K_{u}+1}^{K_{u}+D} \sum_{D=1}^{P_{D}} \mathbf{a}\left(\theta_{k, p}\right) \varsigma_{k, p} s_{k}(t) \\
& +\mathbf{N}(t)
\end{aligned}
$$

which can further be rewritten in a matrix form as

$$
\begin{aligned}
\mathbf{X}(t) & =\mathbf{A}_{u}(\theta, \gamma, \eta) \mathbf{S}_{u}(t)+\mathbf{A}_{c}(\theta, \gamma, \eta) \mathbf{\Gamma} \mathbf{S}_{c}(t)+\mathbf{N}(t) \\
& =\mathbf{A}(\theta, \gamma, \eta) \mathbf{E} \mathbf{S}(t)+\mathbf{N}(t),
\end{aligned}
$$

where $\mathbf{X}(t)=\left[\mathbf{x}_{1}(t), \mathbf{x}_{2}(t), \ldots, \mathbf{x}_{M}(t)\right]^{T}, \mathbf{S}(t)=\left[s_{1}(t), s_{2}(t)\right.$, $\left.\ldots, s_{K}(t)\right]^{T}$, and $\mathbf{N}(t)=\left[\mathbf{n}_{1}(t), \mathbf{n}_{2}(t), \ldots, \mathbf{n}_{M}(t)\right]^{T}$. For brevity, the $\left(K_{u}+D\right) \times 1$ source vector can be described as $\mathbf{S}(t)=$ $\left[\mathbf{S}_{u}^{T}(t), \mathbf{S}_{c}^{T}(t)\right]^{T}$ with $\mathbf{S}_{u}(t)=\left[s_{1}(t), s_{2}(t), \ldots, s_{K_{u}}(t)\right]^{T}$ and $\mathbf{S}_{c}(t)=$ $\left[s_{K_{u}+1}(t), s_{K_{u}+2}(t), \ldots, s_{K_{u}+D}(t)\right]^{T}$ being the source vectors associated with the uncorrelated and coherent sources, respectively. $\mathbf{E}$ is a $K \times\left(K_{u}+D\right)$ block diagonal matrix defined as $\mathbf{E}=\operatorname{blkdiag}\left\{\mathbf{I}_{K u}, \boldsymbol{\Gamma}\right\}$, where $\boldsymbol{\Gamma}$ is the fading coefficient matrix whose $k$ th column is $\varsigma_{k}=\left[\varsigma_{k, 1}, \varsigma_{k, 2}, \ldots\right.$, $\left.\varsigma_{k, p_{k}}\right]^{T}$ for $k=K_{u}+1, \ldots, K_{u}+D$. The $2 M \times K$ array response matrix $\mathbf{A}(\theta, \gamma, \eta)$ is defined as $\mathbf{A}(\theta, \gamma, \eta)=\left[\mathbf{A}_{u}(\theta, \gamma, \eta), \mathbf{A}_{c}(\theta\right.$, $\gamma, \eta)]$, where $\mathbf{A}_{u}(\theta, \gamma, \eta)=\left[\mathbf{a}\left(\theta_{1}, \gamma_{1}, \eta_{1}\right), \mathbf{a}\left(\theta_{2}, \gamma_{2}, \eta_{2}\right), \ldots, \mathbf{a}\left(\theta_{K_{u}}\right.\right.$, $\left.\left.\gamma_{K_{u}}, \eta_{K_{u}}\right)\right]$ corresponds to the $K_{u}$ uncorrelated sources and $\mathbf{A}_{c}(\theta, \gamma, \eta)=\left[\mathbf{A}_{c, K_{u}+1}(\theta, \gamma, \eta), \mathbf{A}_{c, K_{u}+2}(\theta, \gamma, \eta), \ldots, \mathbf{A}_{c, K_{u}+D}(\theta\right.$, $\gamma, \eta)]$ corresponds to the $K_{c}$ coherent sources with $\mathbf{A}_{c, k}(\theta, \gamma, \eta)=\left[\mathbf{a}\left(\theta_{k, 1}, \gamma_{k, 1}, \eta_{k, 1}\right), \mathbf{a}\left(\theta_{k, 2}, \gamma_{k, 2}, \eta_{k, 2}\right), \ldots, \mathbf{a}\left(\theta_{k, p_{k}}\right.\right.$, $\left.\gamma_{k, p_{k}}, \eta_{k, p_{k}}\right)$ ] being the array response matrix with respect to the $k$ th group of coherent sources. The $k$ th column vector of the array response matrix $\mathbf{A}(\theta, \gamma, \eta)$ is defined as

$$
\mathbf{a}\left(\theta_{k}, \gamma_{k}, \eta_{k}\right)=\mathbf{q}\left(\theta_{k}\right) \otimes \mathbf{c}\left(\theta_{k}, \gamma_{k}, \eta_{k}\right),
$$

where the source steering vector $\mathbf{q}\left(\theta_{k}\right)$ and polarization steering vector $\mathbf{c}\left(\theta_{k}, \varphi_{k}, \gamma_{k}\right)$ are, respectively, defined as

$$
\begin{aligned}
& \mathbf{q}\left(\theta_{k}\right)=\left[1, e^{-j 2 \pi d \sin \theta_{k} / \lambda}, \ldots, e^{-j 2 \pi(M-1) d \sin \theta_{k} / \lambda}\right]^{T}, \\
& \mathbf{c}\left(\theta_{k}, \gamma_{k}, \eta_{k}\right)=\left[-\cos \gamma_{k}, \cos \theta_{k} \sin \gamma_{k} e^{j \eta_{k}}\right]^{T} .
\end{aligned}
$$

The objective of the proposed method is to determine the angle and polarization parameters $\left\{\theta_{k}, \gamma_{k}, \eta_{k}, k=1,2, \ldots, K\right\}$ under the condition of coexisting uncorrelated and coherent sources. For notational convenience, $\mathbf{A}(\theta, \gamma, \eta), \mathbf{A}_{u}(\theta, \gamma, \eta)$, $\mathbf{A}_{c}(\theta, \gamma, \eta)$, and $\mathbf{c}\left(\theta_{k}, \gamma_{k}, \eta_{k}\right)$ are, respectively, substituted by $\mathbf{A}$, $\mathbf{A}_{u}, \mathbf{A}_{c}$, and $\mathbf{c}_{k}$ in the following analysis.

The basic assumptions followed throughout this paper are as follows:

(A1) $\mathbf{S}(t)$ and $\mathbf{N}(t)$ are the zero-mean uncorrelated stationary Gaussian random processes that are uncorrelated with each other. Coherent sources $\left\{s_{k}(t)\right\}_{k=K_{u}+1}^{K_{u}+D}$ in different coherent groups are mutually uncorrelated, and they are also uncorrelated with the uncorrelated sources $\left\{s_{k}(t)\right\}_{k=1}^{K_{u}}$.

(A2) The number of uncorrelated and coherent sources, coherent groups, and fading coefficients (i.e., the values of $K_{u}, K_{c}, D$, and $\varsigma_{k, p}$ ) can be estimated by using the source number estimation [24] and the fading coefficients estimation [25].

(A3) To obviate the problem of phase ambiguity, the interelement spacing of the dual-polarization ULA satisfies $d \leq \lambda / 2$, and the number of sources is less than the number of sensors; that is, $K_{u}+D<M$.

\section{Parameter Estimation}

In this section, the angle and polarization estimation method is derived for a mixture of uncorrelated and coherent sources by using a dual-polarization ULA. Based on the modulus property of eigenvalues, the uncorrelated sources are distinguished from the coherent sources. This reduces the mutual interference between uncorrelated and coherent sources and enlarges the effective array aperture. The angle and polarization parameters of each uncorrelated source and that of each coherent group are estimated separately by estimating the corresponding array response matrix, thus eliminating the need of computationally intensive $3 \mathrm{D}$ spectral search. 
3.1. Parameter Estimation for Uncorrelated Sources. The covariance matrix of $\mathbf{X}(t)$ with the size $2 M \times 2 M$ is expressed as

$$
\begin{aligned}
\mathbf{R} & =E\left\{\mathbf{X}(t) \mathbf{X}^{H}(t)\right\}=\mathbf{A E R}_{s} \mathbf{E}^{H} \mathbf{A}^{H}+\sigma_{n}^{2} \mathbf{I} \\
& =\mathbf{A}_{u} \mathbf{R}_{u} \mathbf{A}_{u}^{H}+\mathbf{A}_{c} \boldsymbol{\Gamma} \mathbf{R}_{c} \boldsymbol{\Gamma}^{H} \mathbf{A}_{c}^{H}+\sigma_{n}^{2} \mathbf{I},
\end{aligned}
$$

where the source covariance matrix $\mathbf{R}_{s}=E\left\{\mathbf{S}(t) \mathbf{S}^{H}(t)\right\}$ is segmented into two parts $\mathbf{R}_{s}=\left[\mathbf{R}_{u}, \mathbf{R}_{c}\right]$, where $\mathbf{R}_{u}=$ $E\left\{\mathbf{S}_{u}(t) \mathbf{S}_{u}^{H}(t)\right\}$ and $\mathbf{R}_{c}=E\left\{\mathbf{S}_{c}(t) \mathbf{S}_{c}^{H}(t)\right\}$ are the uncorrelated and coherent source covariance matrices and $\sigma_{n}^{2}$ denotes the noise variance. In the case of $K$ sources comprising $K_{u}$ uncorrelated and $D$ groups of $K_{c}$ coherent sources, $\mathbf{R}_{s}$ is of rank $K_{u}+D$. By applying eigenvalue decomposition (EVD) to $\mathbf{R}$, the source subspace $\mathbf{E}_{s}$ is obtained from the eigenvectors corresponding to the $K_{u}+D$ largest eigenvalues.
It is noted that the columns of $\mathbf{E}_{s}$ and $\mathbf{A E}$ span the same subspace, such that

$$
\mathbf{E}_{s}=\mathbf{A E T}=\left[\mathbf{A}_{u}, \mathbf{A}_{c} \Gamma\right] \mathbf{T}=\left[\begin{array}{cc}
\mathbf{C}_{u} & \mathbf{C}_{c} \Gamma \\
\mathbf{C}_{u} \Delta_{u} & \mathbf{C}_{c} \Delta_{c} \Gamma \\
\vdots & \vdots \\
\mathbf{C}_{u} \Delta_{u}^{M-1} & \mathbf{C}_{c} \Delta_{c}^{M-1} \Gamma
\end{array}\right] \mathbf{T}
$$

where $\mathbf{T}$ is a unique $\left(K_{u}+D\right) \times\left(K_{u}+D\right)$ full rank matrix and $\Delta_{u}=\operatorname{diag}\left\{e^{-j 2 \pi d \sin \theta_{1} / \lambda}, e^{-j 2 \pi d \sin \theta_{2} / \lambda}, \ldots, e^{-j 2 \pi d \sin \theta_{K_{u}} / \lambda}\right\} \in$ $\mathbb{C}^{K_{u} \times K_{u}}$ and $\Delta_{c}=\operatorname{diag}\left\{e^{-j 2 \pi d \sin \theta_{K_{u}+1,1} / \lambda}, \ldots, e^{-j 2 \pi d \sin \theta_{K_{u}+1, p_{1}} / \lambda}\right.$, $\left.\ldots, e^{-j 2 \pi d \sin \theta_{K_{u}+D, 1} / \lambda}, \ldots, e^{-j 2 \pi d \sin \theta_{K_{u}+D, p_{D}} / \lambda}\right\} \in \mathbb{C}^{K_{c} \times K_{c}}$ are the two diagonal matrices that depend only on the arrival-angles. $\mathrm{C}_{u}$ and $\mathrm{C}_{c}$ are, respectively, called the uncorrelated and coherent polarization factor matrices defined by

$$
\begin{aligned}
& \mathbf{C}_{u}=\left[\mathbf{c}_{1}, \mathbf{c}_{2}, \ldots, \mathbf{c}_{K_{u}}\right]=\left[\begin{array}{cccc}
-\cos \gamma_{1} & -\cos \gamma_{2} & \cdots & -\cos \gamma_{K_{u}} \\
\cos \theta_{1} \sin \gamma_{1} e^{j \eta_{1}} & \cos \theta_{2} \sin \gamma_{2} e^{j \eta_{2}} & \cdots & \cos \theta_{K_{u}} \sin \gamma_{K_{u}} e^{j \eta_{K_{u}}}
\end{array}\right] \\
& \mathbf{C}_{c}=\left[\mathbf{c}_{K_{u}+1,1}, \ldots, \mathbf{c}_{K_{u}+1, p_{1}}, \ldots, \mathbf{c}_{K_{u}+D, p_{D}}\right] \\
& =\left[\begin{array}{ccccc}
-\cos \gamma_{K_{u}+1,1} & \cdots & -\cos \gamma_{K_{u}+1, p_{1}} & \cdots & -\cos \gamma_{K_{u}+D, p_{D}} \\
\cos \theta_{K_{u}+1,1} \sin \gamma_{K_{u}+1,1} e^{j \eta_{K_{u}+1,1}} & \cdots & \cos \theta_{K_{u}+1, p_{1}} \sin \gamma_{K_{u}+1, p_{1}} e^{j \eta_{K_{u}+1, p_{1}}} & \cdots & \cos \theta_{K_{u}+D, p_{D}} \sin \gamma_{K_{u}+D, p_{D}} e^{j \eta_{K_{u}+D, p_{D}}}
\end{array}\right] .
\end{aligned}
$$

According to the inherent rotational invariance, $\mathbf{E}_{s}$ can be divided into two $2(M-1) \times\left(K_{u}+D\right)$ overlapped submatrices, where the $l$ th submatrix can be expressed as

$$
\mathbf{E}_{s, l}=\mathbf{J}_{l} \mathbf{E}_{s}
$$

with the selection matrix $\mathbf{J}_{l}$ defined as $\mathbf{J}_{l}=$ $\left[\begin{array}{lll}\mathbf{0}_{2(M-1) \times 2(l-1)} & \mathbf{I}_{2(M-1)} & \mathbf{0}_{2(M-1) \times(4-2 l)}\end{array}\right]$ for $l=1,2$. According to (11) and (14), we have

$$
\mathbf{E}_{s, 1}^{\dagger} \mathbf{E}_{s, 2}=\mathbf{T}^{-1} \Delta \mathbf{T}
$$

where the block diagonal matrix $\Delta$, comprising both uncorrelated and coherent angle information, is expressed as $\Delta=$ blkdiag $\left\{\Delta_{u}, \Gamma^{\dagger} \Delta_{c} \boldsymbol{\Gamma}\right\}$. As can be seen in (15), the diagonal matrix $\Delta$ is composed of $K_{u}+D$ eigenvalues of the matrix
$\mathbf{E}_{s, 1}^{\dagger} \mathbf{E}_{s, 2}$, and the full rank matrix $\mathbf{T}^{-1}$ is composed of the corresponding eigenvectors of matrix $\mathbf{E}_{s, 1}^{\dagger} \mathbf{E}_{s, 2}$. Based on the modulus property outlined in [26], the moduli of the elements in $\Delta$, corresponding to uncorrelated sources, are approximately equivalent to 1 in the case of noise disturbance, while those corresponding to coherent groups are significantly away from 1. As a result, the uncorrelated sources are distinguished from coherent sources, and the angles of uncorrelated sources are thereby resolved.

$K_{u}$ columns of $\mathbf{T}^{-1}$ corresponding to $K_{u}$ uncorrelated sources are extracted in order to construct the matrix $\mathbf{T}_{u}^{-1}$. Similarly, the remaining $D$ columns, corresponding to $D$ coherent groups, are chosen from $\mathbf{T}^{-1}$ to construct matrix $\mathbf{T}_{c}^{-1}$. According to (11), the estimation of $\mathbf{A}_{u}$ is defined as

$$
\widehat{\mathbf{A}}_{u}=\mathbf{E}_{s} \mathbf{T}_{u}^{-1}=\left[\begin{array}{c}
\mathbf{C}_{u} \\
\mathbf{C}_{u} \Delta_{u} \\
\vdots \\
\mathbf{C}_{u} \Delta_{u}^{M-1}
\end{array}\right]=\left[\begin{array}{ccc}
-\cos \gamma_{1} & \cdots & -\cos \gamma_{K_{u}} \\
\cos \theta_{1} \sin \gamma_{1} e^{j \eta_{1}} & \cdots & \cos \theta_{K_{u}} \sin \gamma_{K_{u}} e^{j \eta_{K_{u}}} \\
-\cos \gamma_{1} e^{-j 2 \pi d \sin \theta_{1} / \lambda} & \cdots & -\cos \gamma_{K_{u}} e^{-j 2 \pi d \sin \theta_{K_{u}} / \lambda} \\
\cos \theta_{1} \sin \gamma_{1} e^{-j 2 \pi d \sin \theta_{1} / \lambda} & \cdots & \cos \theta_{K_{u}} \sin \gamma_{K_{u}} e^{j \eta_{K_{u}}} e^{-j 2 \pi d \sin \theta_{K_{u}} / \lambda} \\
\vdots & \ddots & \vdots \\
-\cos \gamma_{1} e^{-j 2 \pi(M-1) d \sin \theta_{1} / \lambda} & \cdots & -\cos \gamma_{K_{u}} e^{-j 2 \pi(M-1) d \sin \theta_{K_{u}} / \lambda} \\
\cos \theta_{1} \sin \gamma_{1} e^{-j 2 \pi(M-1) d \sin \theta_{1} / \lambda} & \cdots & \cos \theta_{K_{u}} \sin \gamma_{K_{u}} e^{j \eta_{K_{u}}} e^{-j 2 \pi(M-1) d \sin \theta_{K_{u}} / \lambda}
\end{array}\right] .
$$


For the $k$ th $\left(k=1,2, \ldots, K_{u}\right)$ uncorrelated source, the relevant array response matrix $\widehat{\mathbf{A}}_{u, k}$ is formulated on similar lines as for (16); that is, $\widehat{\mathbf{A}}_{u, k}=\mathbf{E}_{s} \mathbf{T}_{u, k}^{-1}$, where $\mathbf{T}_{u, k}^{-1}$ is the eigenvector of the $k$ th uncorrelated source extracted from $\mathbf{T}_{u}^{-1}$. Further, the corresponding auxiliary polarization angle $\widehat{\gamma}_{k}$ and the polarization phase difference $\widehat{\eta}_{k}$ of the $k$ th uncorrelated source are, respectively, obtained as

$$
\begin{aligned}
\widehat{\gamma}_{k} & =\frac{1}{M} \\
& \cdot \sum_{i=1}^{M} \tan ^{-1}\left(\left|\widehat{\mathbf{A}}_{u, k}(2 i, k)\right| \cdot /\left(\left|\widehat{\mathbf{A}}_{u, k}(2 i-1, k)\right| \cos \theta_{k}\right)\right), \\
\widehat{\eta}_{k} & =\frac{1}{M} \sum_{i=1}^{M} \arg \left(\widehat{\mathbf{A}}_{u, k}(2 i, k) \cdot / \widehat{\mathbf{A}}_{u, k}(2 i-1, k)\right) .
\end{aligned}
$$

It can be observed from (17) and (18) that the polarization parameters of the uncorrelated sources are estimated by taking advantages of the inherent structure of $\widehat{\mathbf{A}}_{u, k}$, which is easy to implement by using elementwise division operation. In addition, the proposed method accomplishes the estimation of angle and polarization parameters for the uncorrelated sources effectively without resorting to parameter matching.

3.2. Parameter Estimation for Coherent Sources. Similar to the estimation of $\mathbf{A}_{u}$ outlined in Section 3.1, the array response matrix of the $k$ th $\left(k=K_{u}+1, K_{u}+2, \ldots, K_{u}+D\right)$ coherent group $\mathbf{A}_{c, k}$ can be estimated as

$$
\widehat{\mathbf{A}}_{c, k}=\mathbf{E}_{s} \mathbf{T}_{c, k}^{-1}=\left[\begin{array}{c}
\mathbf{C}_{c, k} \boldsymbol{\varsigma}_{k} \\
\mathbf{C}_{c, k} \boldsymbol{\Delta}_{c, k} \boldsymbol{\varsigma}_{k} \\
\vdots \\
\mathbf{C}_{c, k} \Delta_{c, k}^{M-1} \boldsymbol{\varsigma}_{k}
\end{array}\right] .
$$

This can further be rewritten in an extensive form as

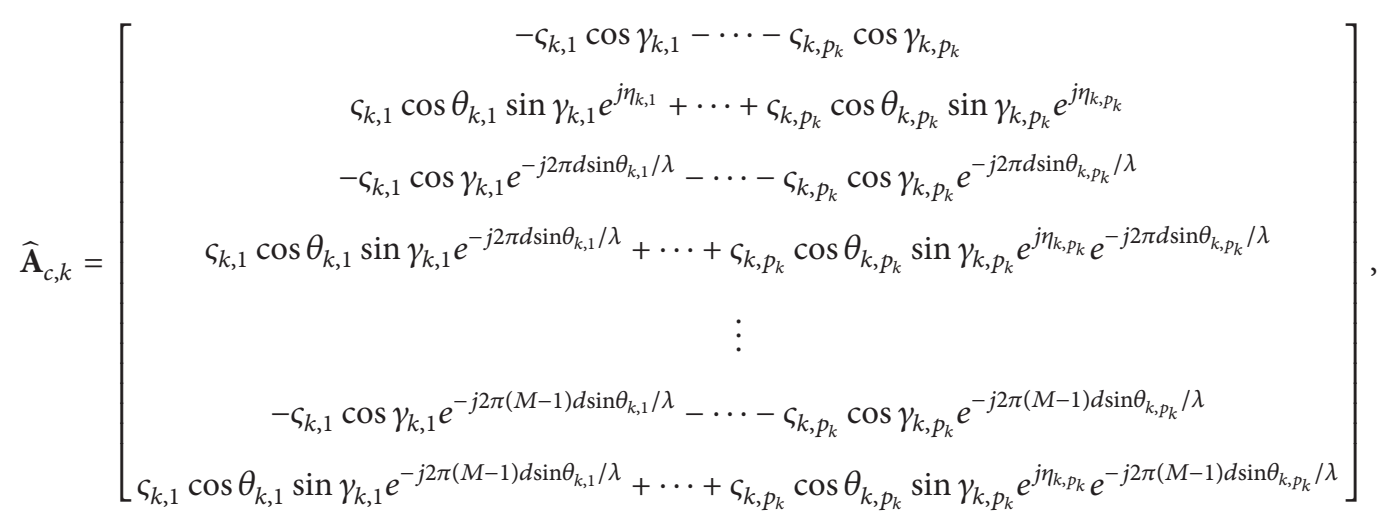

where $\mathbf{C}_{c, k}=\left[\mathbf{c}_{k, 1}, \mathbf{c}_{k, 2}, \ldots, \mathbf{c}_{k, p_{k}}\right]$ and $\boldsymbol{\Delta}_{c, k}=$ $\operatorname{diag}\left\{e^{-j 2 \pi d \sin \theta_{k, 1} / \lambda}, \ldots, e^{-j 2 \pi d \sin \theta_{k, p_{k}} / \lambda}\right\}$. Note that the odd and even rows of $\widehat{\mathbf{A}}_{c, k}$ characterize the array response submatrix of $x$ - and $y$-axes components of the dual-polarization vector sensor array, respectively. Thus, we divide $\widehat{\mathbf{A}}_{c, k}$ of size $2 M \times 1$ into two $M \times 1$ submatrices using the following equation:

$$
\widehat{\mathbf{A}}_{c, k}^{(i)}=\mathbf{G}_{i}^{T} \widehat{\mathbf{A}}_{c, k}, \quad i=1,2
$$

such that $\mathbf{G}_{1}$ and $\mathbf{G}_{2}$ are two exchange matrices, defined as

$$
\begin{aligned}
& \mathbf{G}_{1}=\left[\mathbf{g}_{1}, \mathbf{g}_{3}, \ldots, \mathbf{g}_{2 M-1}\right], \\
& \mathbf{G}_{2}=\left[\mathbf{g}_{2}, \mathbf{g}_{4}, \ldots, \mathbf{g}_{2 M}\right],
\end{aligned}
$$

where $\mathbf{g}_{i}$ is a $2 M \times 1$ vector having the element on the $i$ th row as one and the remaining elements as zeros. For the purpose of "decorrelation," the two Hankel matrices constructed by the array response matrices of $x$ - and $y$-axes components with respect to the $k$ th coherent group are given as

$$
\begin{aligned}
& \mathbf{B}_{k}^{(1)} \\
& =\left[\begin{array}{cccc}
\widehat{\mathbf{A}}_{c, k}^{(1)}(1) & \widehat{\mathbf{A}}_{c, k}^{(1)}(2) & \cdots & \widehat{\mathbf{A}}_{c, k}^{(1)}\left(p_{k}+1\right) \\
\widehat{\mathbf{A}}_{c, k}^{(1)}(2) & \widehat{\mathbf{A}}_{c, k}^{(1)}(3) & \cdots & \widehat{\mathbf{A}}_{c, k}^{(1)}\left(p_{k}+2\right) \\
\vdots & \vdots & \ddots & \vdots \\
\widehat{\mathbf{A}}_{c, k}^{(1)}\left(M-p_{k}\right) & \widehat{\mathbf{A}}_{c, k}^{(1)}\left(M-p_{k}+1\right) & \cdots & \widehat{\mathbf{A}}_{c, k}^{(1)}(M)
\end{array}\right] \\
& \mathbf{B}_{k}^{(2)} \\
& =\left[\begin{array}{cccc}
\widehat{\mathbf{A}}_{c, k}^{(2)}(1) & \widehat{\mathbf{A}}_{c, k}^{(2)}(2) & \cdots & \widehat{\mathbf{A}}_{c, k}^{(2)}\left(p_{k}+1\right) \\
\widehat{\mathbf{A}}_{c, k}^{(2)}(2) & \widehat{\mathbf{A}}_{c, k}^{(2)}(3) & \cdots & \widehat{\mathbf{A}}_{c, k}^{(2)}\left(p_{k}+2\right) \\
\vdots & \vdots & \ddots & \vdots \\
\widehat{\mathbf{A}}_{c, k}^{(2)}\left(M-p_{k}\right) & \widehat{\mathbf{A}}_{c, k}^{(2)}\left(M-p_{k}+1\right) & \cdots & \widehat{\mathbf{A}}_{c, k}^{(2)}(M)
\end{array}\right],
\end{aligned}
$$

where $M-p_{k}>p_{k}$. It is easily proven that the two matrices $\mathbf{B}_{k}^{(1)}$ and $\mathbf{B}_{k}^{(2)}$ have a full rank and can be further expressed as 


$$
\begin{aligned}
& \mathbf{B}_{k}^{(1)} \\
& =\left[\begin{array}{cccc}
\mathbf{C}_{c_{k, 1}} \boldsymbol{\varsigma}_{k} & \mathbf{C}_{c_{k, 1}} \Delta_{c, k} \boldsymbol{\varsigma}_{k} & \cdots & \mathbf{C}_{c_{k, 1}} \Delta_{c, k}^{p_{k}} \boldsymbol{\varsigma}_{k} \\
\mathbf{C}_{c_{k, 1}} \Delta_{c, k} \boldsymbol{\varsigma}_{k} & \mathbf{C}_{c_{k, 1}} \Delta_{c, k}^{2} \boldsymbol{s}_{k} & \cdots & \mathbf{C}_{c_{k, 1}} \Delta_{c, k}^{p_{k}+1} \boldsymbol{\varsigma}_{k} \\
\vdots & \vdots & \ddots & \vdots \\
\mathbf{C}_{c_{k, 1}} \Delta_{c, k}^{M-p_{k}-1} \boldsymbol{\varsigma}_{k} & \mathbf{C}_{c_{k, 1}} \Delta_{c, k}^{M-p_{k}} \boldsymbol{s}_{k} & \cdots & \mathbf{C}_{\mathcal{c}_{k, 1}} \Delta_{c, k}^{M-1} \boldsymbol{\varsigma}_{k}
\end{array}\right], \\
& \mathbf{B}_{k}^{(2)} \\
& =\left[\begin{array}{cccc}
\mathbf{C}_{c_{k, 2}} \boldsymbol{\varsigma}_{k} & \mathbf{C}_{c_{k, 2}} \Delta_{c, k} \boldsymbol{\varsigma}_{k} & \cdots & \mathbf{C}_{c_{k, 2}} \Delta_{c, k}^{p_{k}} \boldsymbol{\varsigma}_{k} \\
\mathbf{C}_{c_{k, 2}} \Delta_{c, k} \boldsymbol{\varsigma}_{k} & \mathbf{C}_{c_{k, 2}} \Delta_{c, k}^{2} \boldsymbol{\varsigma}_{k} & \cdots & \mathbf{C}_{c_{k, 2}} \Delta_{c, k}^{p_{k}+1} \boldsymbol{\varsigma}_{k} \\
\vdots & \vdots & \ddots & \vdots \\
\mathbf{C}_{c_{k, 2}} \Delta_{c, k}^{M-p_{k}-1} \boldsymbol{\varsigma}_{k} & \mathbf{C}_{c_{k, 2}} \Delta_{c, k}^{M-p_{k}} \boldsymbol{\varsigma}_{k} & \cdots & \mathbf{C}_{c_{k, 2}} \Delta_{c, k}^{M-1} \boldsymbol{\varsigma}_{k}
\end{array}\right],
\end{aligned}
$$

where $\mathbf{C}_{c_{k, 1}}$ and $\mathbf{C}_{c_{k, 2}}$ are, respectively, composed of the first and the second rows of $\mathbf{C}_{c, k}$. The matrix $\Delta_{c, k}=$ $\operatorname{diag}\left\{e^{-j 2 \pi d \sin \theta_{k, 1} / \lambda}, \ldots, e^{-j 2 \pi d \sin \theta_{k, p_{k}} / \lambda}\right\}$ corresponding to the $k$ th $\left(k=K_{u}+1, \ldots, K_{u}+D\right)$ coherent group can be extracted from $\Delta_{c}$. In view of the fact that $\mathbf{B}_{k}^{(1)}$ and $\mathbf{B}_{k}^{(2)}$ have the same rotational-invariant structures, either of them can be utilized for the estimation of coherent angles. In this study, $\mathbf{B}_{k}^{(1)}$ is taken as an example. First, two rotational-invariant submatrix pairs are constructed: the first pair is $\mathbf{B}_{k, r_{1}}^{(1)}=\mathbf{B}_{k}^{(1)}(1: M-$ $\left.p_{k}-1,:\right)$ and $\mathbf{B}_{k, r_{2}}^{(1)}=\mathbf{B}_{k}^{(1)}\left(2: M-p_{k},:\right)$; the second pair is $\mathbf{B}_{k, l_{1}}^{(1)}=\mathbf{B}_{k}^{(1)}\left(:, 1: M-p_{k}-1\right)$ and $\mathbf{B}_{k, l_{2}}^{(1)}=\mathbf{B}_{k}^{(1)}(:, 2: M-$ $\left.p_{k}\right)$. Subsequently, the rotational invariance can be applied to the two submatrix pairs twice, which results in two groups of coherent angle estimation results $\left\{\widehat{\theta}_{k, 1}^{1}, \widehat{\theta}_{k, 2}^{1}, \ldots, \widehat{\theta}_{k, p_{k}}^{1}\right\}$ and $\left\{\hat{\theta}_{k, 1}^{2}, \widehat{\theta}_{k, 2}^{2}, \ldots, \widehat{\theta}_{k, p_{k}}^{2}\right\}$. The final coherent angle estimation is then achieved as $\left\{\widehat{\theta}_{k, 1}, \widehat{\theta}_{k, 2}, \ldots, \widehat{\theta}_{k, p_{k}}\right\}=\left\{\left(\hat{\theta}_{k, 1}^{1}+\widehat{\theta}_{k, 1}^{2}\right) / 2,\left(\hat{\theta}_{k, 2}^{1}+\right.\right.$ $\left.\left.\widehat{\theta}_{k, 2}^{2}\right) / 2, \ldots,\left(\hat{\theta}_{k, p_{k}}^{1}+\widehat{\theta}_{k, p_{k}}^{2}\right) / 2\right\}$ that allows more accurate angle estimation than the conventional ESPRIT method along with a slight computational gain.

For further coherent polarization estimation, $\mathbf{B}_{k}^{(1)}$ and $\mathbf{B}_{k}^{(2)}$ can be rewritten as

$$
\begin{aligned}
\mathbf{B}_{k}^{(1)} & =\left[\begin{array}{c}
\mathbf{C}_{c_{k, 1}} \\
\mathbf{C}_{c_{k, 1}} \Delta_{c, k} \\
\vdots \\
\mathbf{C}_{c_{k, 1}} \Delta_{c, k}^{M-p_{k}-1}
\end{array}\right]\left[\begin{array}{llll}
\boldsymbol{\varsigma}_{k} & \Delta_{c, k} \boldsymbol{\varsigma}_{k} & \cdots & \Delta_{c, k}^{p_{k}} \boldsymbol{\varsigma}_{k}
\end{array}\right], \\
\mathbf{B}_{k}^{(2)} & =\left[\begin{array}{c}
\mathbf{C}_{c_{k, 2}} \\
\mathbf{C}_{c_{k, 2}} \Delta_{c, k} \\
\vdots \\
\mathbf{C}_{c_{k, 2}} \Delta_{c, k}^{M-p_{k}-1}
\end{array}\right]\left[\begin{array}{llll}
\boldsymbol{\varsigma}_{k} & \Delta_{c, k} \boldsymbol{\varsigma}_{k} & \cdots & \Delta_{c, k}^{p_{k}} \boldsymbol{\varsigma}_{k}
\end{array}\right] .
\end{aligned}
$$

In order to determine the polarization parameters of the coherent sources, the theory of least-squares is adopted such that

$$
\Delta \mathbf{Q}_{k}=\min \left\|\mathbf{B}_{k}^{(1)} \mathbf{Q}_{k}-\mathbf{B}_{k}^{(2)}\right\|_{F}^{2},
$$

where (29) is referred to as the coherent polarization equation. The derivative of $\Delta \mathbf{Q}_{k}$ with respect to the coherent polarization matrix $\mathbf{Q}_{k}$ is defined as

$$
\begin{aligned}
\frac{\partial \Delta \mathbf{Q}_{k}}{\partial \mathbf{Q}_{k}}= & -\left(\mathbf{B}_{k}^{(2)}\right)^{T} \mathbf{B}_{k}^{(1)}-\left(\mathbf{B}_{k}^{(1)}\right)^{T} \mathbf{B}_{k}^{(2)} \\
& +\left(\mathbf{B}_{k}^{(1)}\right)^{T} \mathbf{B}_{k}^{(1)} \mathbf{Q}_{k}+\mathbf{Q}_{k}^{T}\left(\mathbf{B}_{k}^{(1)}\right)^{T} \mathbf{B}_{k}^{(1)} .
\end{aligned}
$$

The solution to (29) is acquired by setting the equality in (30) to be zero. Thus, we have

$$
\mathbf{Q}_{k}=\left(\left(\mathbf{B}_{k}^{(1)}\right)^{T} \mathbf{B}_{k}^{(1)}\right)^{-1}\left(\mathbf{B}_{k}^{(1)}\right)^{T} \mathbf{B}_{k}^{(2)}=\left(\mathbf{B}_{k}^{(1)}\right)^{\dagger} \mathbf{B}_{k}^{(2)} .
$$

By substituting (27) and (28) into (31), $\mathbf{Q}_{k}$ can be further reformulated as (see Appendix for details)

$\mathbf{Q}_{k}$

$$
\begin{aligned}
& =\left(\left[\begin{array}{c}
\mathbf{C}_{c_{k, 1}} \\
\mathbf{C}_{c_{k, 1}} \Delta_{c, k} \\
\vdots \\
\mathbf{C}_{c_{k, 1}} \Delta_{c, k}^{M-p_{k}-1}
\end{array}\right]\left[\begin{array}{llll}
\boldsymbol{\varsigma}_{k} & \Delta_{c, k} \boldsymbol{\varsigma}_{k} & \cdots & \Delta_{c, k}^{p_{k}} \boldsymbol{\varsigma}_{k}
\end{array}\right]\right)^{\dagger} \\
& \left(\left[\begin{array}{c}
\mathbf{C}_{c_{k, 2}} \\
\mathbf{C}_{c_{k, 2}} \Delta_{c, k} \\
\vdots \\
\mathbf{C}_{c_{k, 2}} \Delta_{c, k}^{M-p_{k}-1}
\end{array}\right]\left[\begin{array}{llll}
\boldsymbol{\varsigma}_{k} & \Delta_{c, k} \boldsymbol{\varsigma}_{k} & \cdots & \Delta_{c, k}^{p_{k}} \boldsymbol{\varsigma}_{k}
\end{array}\right]\right) \\
& =\left[\begin{array}{llll}
\boldsymbol{\varsigma}_{k} & \boldsymbol{\Delta}_{c, k} \boldsymbol{\varsigma}_{k} & \cdots & \Delta_{c, k}^{p_{k}} \boldsymbol{\varsigma}_{k}
\end{array}\right]^{\dagger} \\
& {\left[\begin{array}{cccc}
\mu_{k, 1} & & & \\
& \mu_{k, 2} & & \\
& & \ddots & \\
& & & \mu_{k, p_{k}}
\end{array}\right]\left[\begin{array}{llll}
\boldsymbol{\varsigma}_{k} & \Delta_{c, k} \boldsymbol{\varsigma}_{k} & \cdots & \Delta_{c, k}^{p_{k}} \boldsymbol{\varsigma}_{k}
\end{array}\right]}
\end{aligned}
$$

where $\mu_{k, i}=-\tan \gamma_{k, i} \cos \theta_{k, i} e^{j \eta_{k, i}}, i=1,2, \ldots, p_{k}$. Equation (32) indicates that the polarization information related to the $k$ th group coherent source can be obtained from the eigenvalues of $\mathbf{Q}_{k}$. Thus, the estimations of polarization phase difference are given by

$$
\begin{aligned}
\widehat{\eta}_{k, i}= & -\arg \left(\mu_{k, i}\right), \\
& k=K_{u}+1, K_{u}+2, \ldots, K_{u}+D, i=1,2, \ldots, p_{k} .
\end{aligned}
$$

To achieve the estimation of coherent auxiliary polarization angles, the associated angle and polarization phase 
differences must obey a one-to-one relationship. For this purpose, we resort to a simple pair-matching method outlined in [14]. The matching method is implemented via a simple eigenvalue-matching operation, which is effective at the cost of a negligible increase in computational cost. The estimated auxiliary polarization angles are obtained as

$$
\begin{aligned}
\widehat{\gamma}_{k, i}= & \tan ^{-1}\left(\frac{\left|\mu_{k, i}\right|}{\cos \theta_{k, i}}\right), \\
& k=K_{u}+1, K_{u}+2, \ldots, K_{u}+D, i=1,2, \ldots, p_{k} .
\end{aligned}
$$

The estimation of joint angle and polarization is therefore accomplished using the proposed method under the coexistence of both uncorrelated and coherent sources. The major steps are summarized as follows.

(1) Calculate the array covariance matrix $\mathbf{R}$ in (10) via the sample estimation $\widehat{\mathbf{R}}=\sum_{n=1}^{N} \mathbf{X}\left(t_{n}\right) \mathbf{X}^{H}\left(t_{n}\right) / N$, where $N$ is the snapshot number.

(2) Based on the moduli property of the eigenvalues, the uncorrelated sources are firstly separated from the coherent sources, and the uncorrelated angle parameters are then estimated by exploiting the rotationalinvariance properties according to (11), (14), and (15). Subsequently, the associated auxiliary polarization angle and the polarization phase difference are estimated by (17) and (18).

(3) The coherent array response matrix is estimated according to (19)-(22) and is then divided into two submatrices $\widehat{\mathbf{A}}_{c, k}^{(1)}$ and $\widehat{\mathbf{A}}_{c, k}^{(2)}$. Further, two Hankel matrices $\mathbf{B}_{k}^{(1)}$ and $\mathbf{B}_{k}^{(2)}$ are constructed for "decorrelating" via (23)-(26), and the estimation of coherent angles is obtained by utilizing two rotational-invariant submatrix pairs. On the basis of the least-square theory, the estimations of coherent auxiliary polarization angle and the polarization phase difference are obtained from (29)-(34).

\section{Discussion}

To provide insights into the proposed method, its identifiability and the computational complexity are discussed in this section.

4.1. Identifiability. Consider $K$ far-field completely polarized narrowband sources composed of $K_{u}$ uncorrelated sources and $D$ groups of $K_{c}$ coherent sources impinging upon the dual-polarization ULA, as described in Section 2. For the parameter estimation of the uncorrelated sources mentioned in Section 3.1, the inequality $M-1>K_{u}+D$ must hold true in the light of (15), thus requiring at least $K_{u}+D+2$ dualpolarization sensors. In addition, to estimate the angle and polarization parameters of the coherent sources according to Section 3.2, the dimensions of the Hankel matrices $\mathbf{B}_{k}^{(1)}$ and $\mathbf{B}_{k}^{(2)}$ defined in (23) and (24) determine the minimum number of dual-polarization sensors required for the coherent parameter estimation. Note that the inequality $M-p_{k}>p_{k}+1$ must be satisfied, such that the minimum number of the dual-polarization sensors required for the coherent sources is $\max \left\{2 p_{1}+1,2 p_{2}+1, \ldots, 2 p_{D}+1\right\}$. Based on the above analysis, it is clear that the proposed method necessitates at least $M=\max \left\{K_{u}+D+2, \max \left\{2 p_{1}+1,2 p_{2}+1, \ldots, 2 p_{D}+1\right\}\right\}$ dual-polarization sensors to resolve all the incident sources, which implies that the proposed method displays potential to estimate more sources than the number of sensors.

4.2. Computational Complexity. Unlike the IPAS method [21], the proposed method distinguishes the uncorrelated sources from the coherent sources by directly utilizing the modulus property of eigenvalues without resorting to the extra operations, such as use of oblique projection and spatial difference matrices; thus the computational complexity of the proposed method is reduced. The major computational load of the proposed method and the IPAS method is to perform the EVD. The EVD requires about $O\left(M^{3}\right)$ computations for the uncorrelated sources and $O\left(\left(p_{k}+1\right)^{3}\right)$ for the coherent sources, which is relatively low as compared with the requirement for IPAS method $\left(O\left(M^{3}\right)\right.$ for uncorrelated sources and $O\left(M^{3}\right)$ for coherent sources). For the existing "decorrelation" methods, such as PS-MUSIC, PDS-MUSIC, and PAS methods, a computationally intensive spectral search is involved; thus the computational complexities of these methods are relative high, especially for the finer spectral search. In summary, the proposed method offers a significant advantage in terms of computational efficiency.

\section{Simulation Results}

In this section, several simulations are presented to illustrate the performance of the proposed method in terms of angle and polarization estimation, where the PAS [20], IPAS [21], PS-MUSIC [17], PDS-MUSIC [18], and the well-known polarized MUSIC methods [9] are selected for comparative characterization.

In the first simulation, we illustrate the effectiveness of the proposed method in distinguishing the uncorrelated sources from the coherent sources and in estimating the joint angle and polarization. A 12-sensor dual-polarization ULA is utilized to this effect. Assume that five far-field completely polarized narrowband electromagnetic wave sources, comprising the two uncorrelated sources and a group of three coherent sources, impinge on this ULA. The uncorrelated sources are parameterized by $\left\{67.3^{\circ}, 27^{\circ}, 14^{\circ}\right\}$ and $\left\{42.9^{\circ}, 19^{\circ}, 50^{\circ}\right\}$, and the coherent sources are parameterized by $\left\{25.7^{\circ}, 40^{\circ}, 16^{\circ}\right\}$, $\left\{-10.1^{\circ}, 13^{\circ}, 30^{\circ}\right\}$, and $\left\{15.4^{\circ}, 23^{\circ}, 82^{\circ}\right\}$ with the fading coefficients $[1,-0.5280+0.6010 j, 0.9335-0.3585 j]$. The SNR and the snapshot number are, respectively, set to be $20 \mathrm{~dB}$ and 500. Figure 2 depicts the moduli of eigenvalues for both uncorrelated and coherent sources. The scatter plots of the uncorrelated and coherent estimations with 100 independent trials are, respectively, shown in Figures 3 and 4 .

It can be clearly observed in Figure 2 that the moduli of the eigenvalues associated with the uncorrelated sources are close to 1 , while that associated with the coherent group is significantly away from 1 . Thus, uncorrelated sources can be effectively distinguished from the coherent sources on 


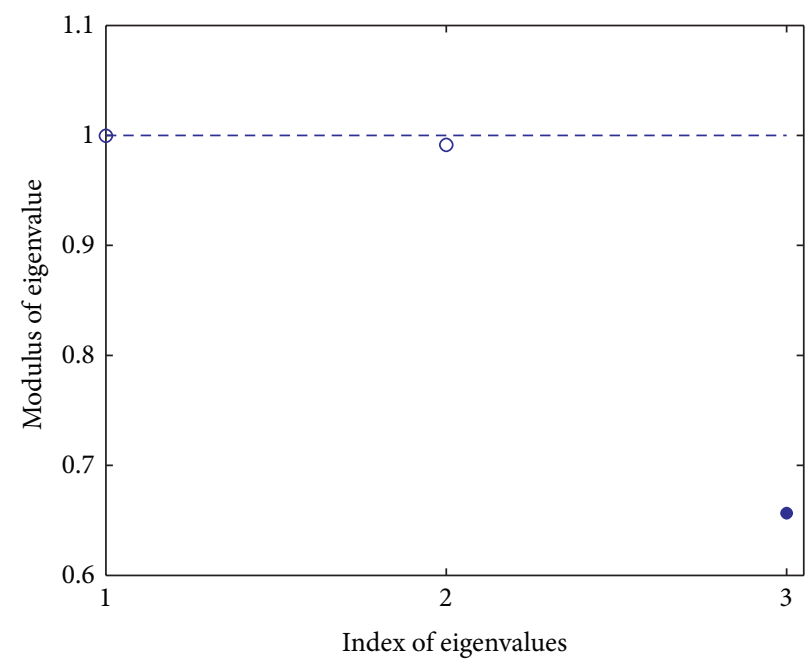

- Uncorrelated sources

- Coherent sources

FIGURE 2: Moduli of the eigenvalues.

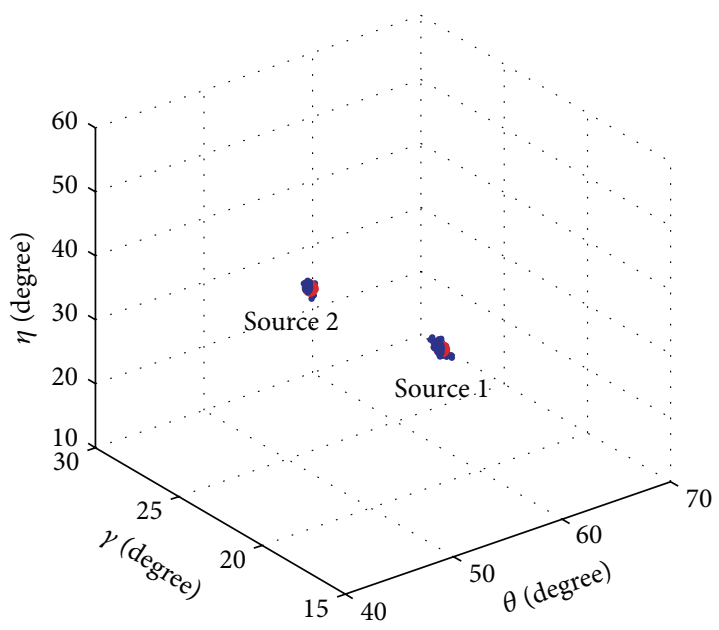

- Estimations

Incident sources

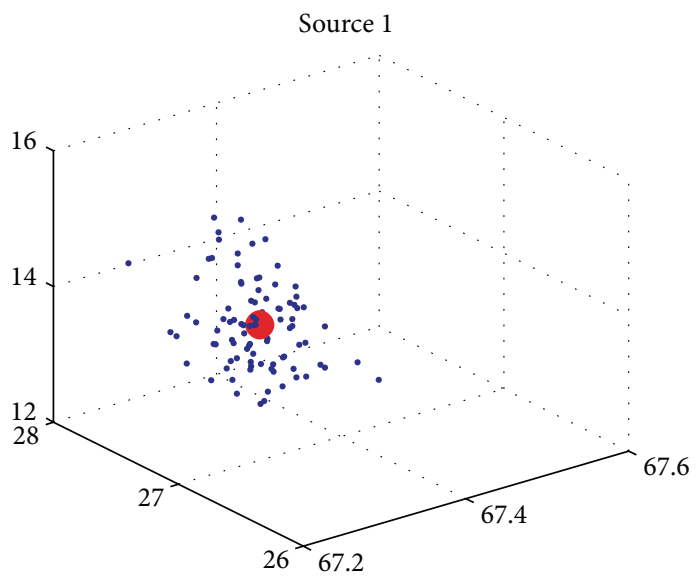

- Estimations

Incident sources

(a)

Source 2

(b)

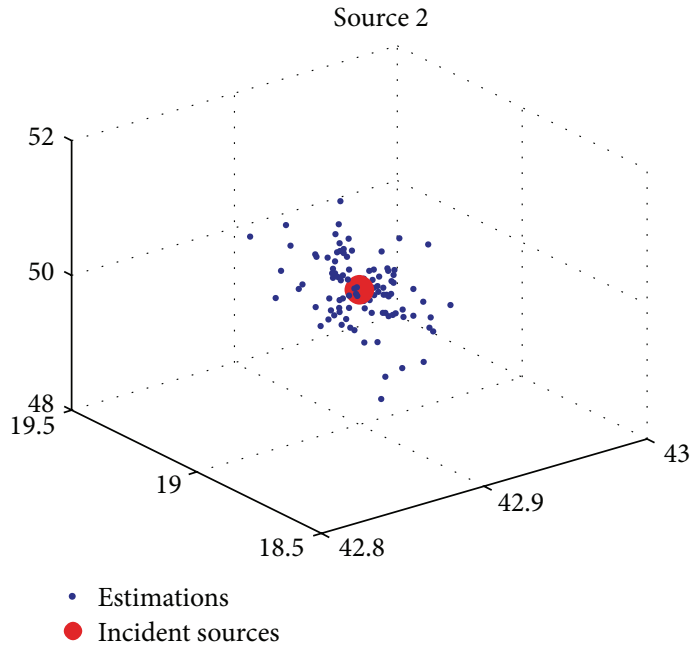

(c)

FiguRE 3: Scatter plot of the uncorrelated sources: (a) global figure; (b) enlarged local scatter plot for the uncorrelated source 1; (c) enlarged local scatter plot for uncorrelated source 2 . 


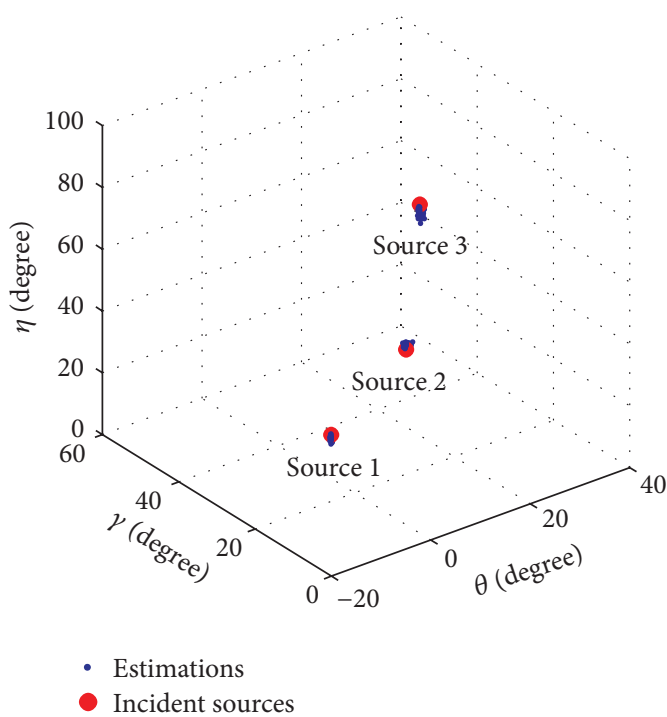

(a)

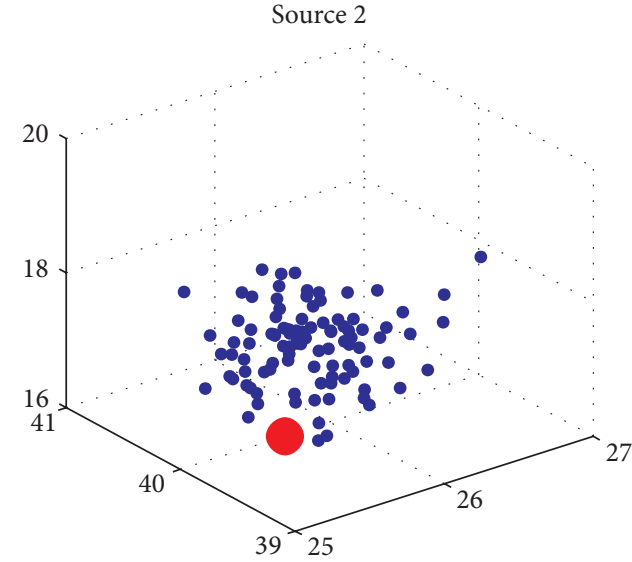

- Estimations

- Incident sources

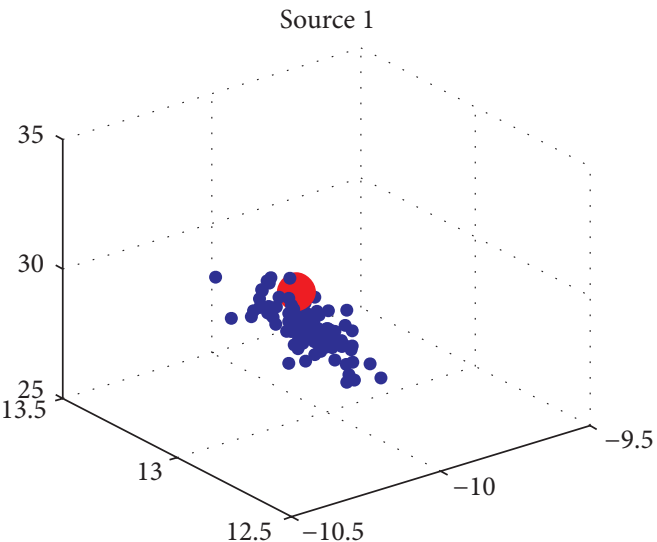

- Estimations

- Incident sources

Source 3

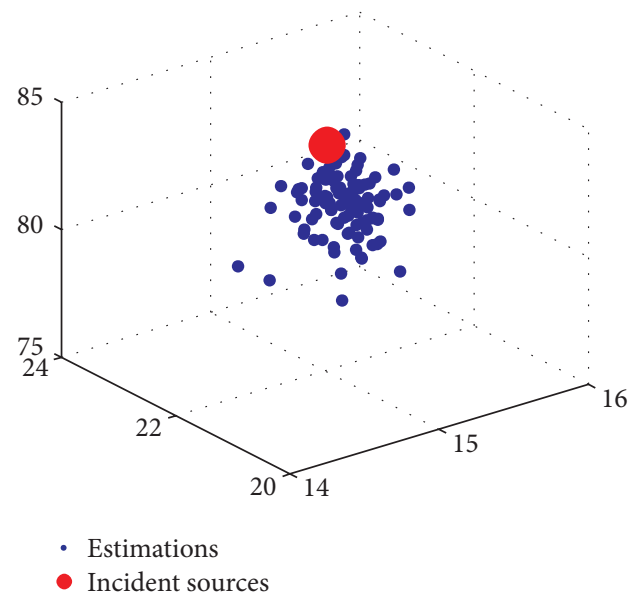

(d)

FiguRE 4: Scatter plot of the coherent sources. (a) Global figure; (b), (c), and (d) display the enlarged local scatter plots for coherent sources 1,2 , and 3 , respectively.

the basis of this modulus property. The results in Figures 3 and 4 demonstrate that the proposed method can accurately estimate the angle and polarization parameters for a mixture of uncorrelated and coherent sources.

In order to evaluate the angle and polarization estimation performance of the proposed method, 200 independent Monte Carlo trials are performed, and the root mean squared error (RMSE) is chosen as a performance index, which is defined as

$$
\operatorname{RMSE}=\sqrt{\frac{1}{200 \widetilde{K}} \sum_{n=1}^{200} \sum_{k=1}^{\widetilde{K}}\left(\widehat{\omega}_{n, k}-\omega_{n, k}\right)^{2}},
$$

where $\widehat{\omega}_{n, k}$ is the estimate of angle or polarization parameter $\omega_{n, k}$ for the $k$ th Monte Carlo trial and $\widetilde{K}$ denotes the number of uncorrelated or coherent sources.
The second simulation studies the performance of the proposed method in estimating the angle and polarization parameters for both the uncorrelated and coherent sources using an 8-sensor dual-polarization ULA. The uncorrelated sources are parameterized by $\left\{82.8^{\circ}, 27^{\circ}, 40^{\circ}\right\}$ and $\left\{56.4^{\circ}, 9^{\circ}, 78^{\circ}\right\}$, and the two coherent sources are parameterized by $\left\{31.7^{\circ}, 30^{\circ}, 16^{\circ}\right\}$ and $\left\{8.0^{\circ}, 13^{\circ}, 30^{\circ}\right\}$ with the fading coefficients $[1,0.4469-0.7696 j]$. Figure 5 shows that the proposed method can effectively estimate the parameters of the uncorrelated and the coherent sources, and the accuracy of the proposed method tends to improve with an increase of SNR or number of snapshots.

The third simulation compares the estimation performance of the proposed method with that of the PAS and the IPAS methods. An 8-sensor dual-polarization ULA is utilized for the proposed method, and an L-shape array composed of eight 6-component electromagnetic vector 


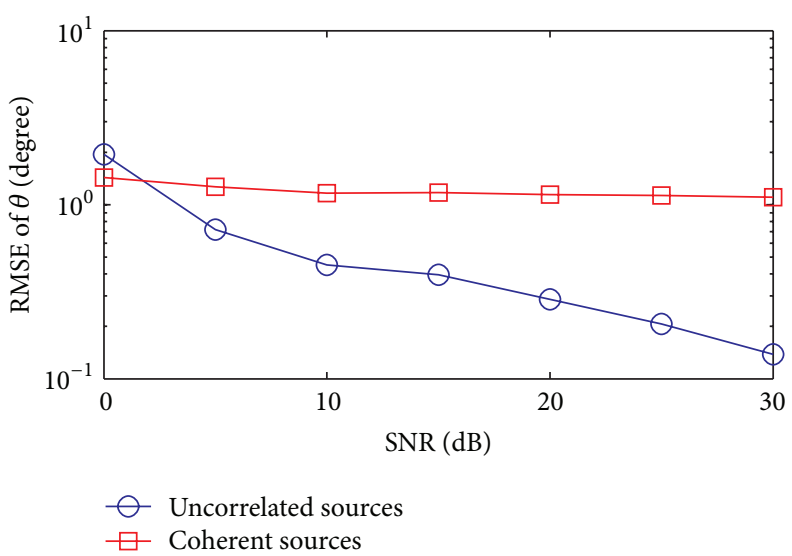

(a)

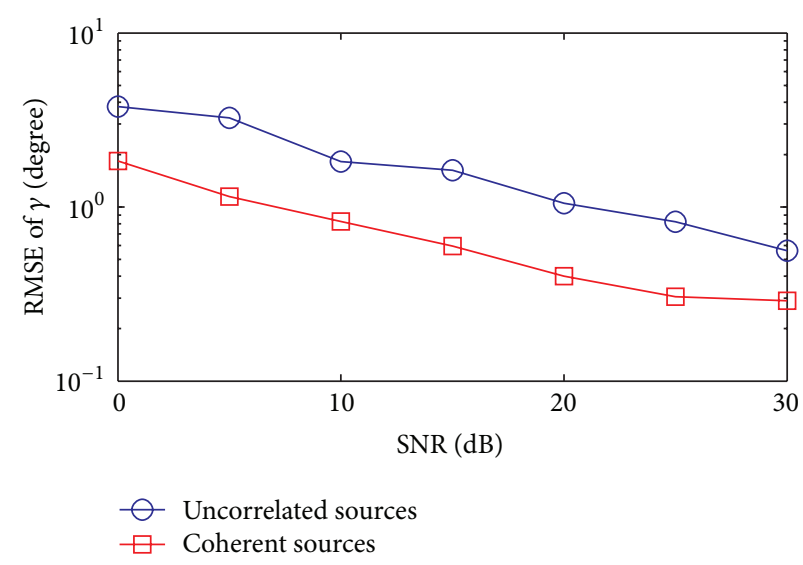

(c)

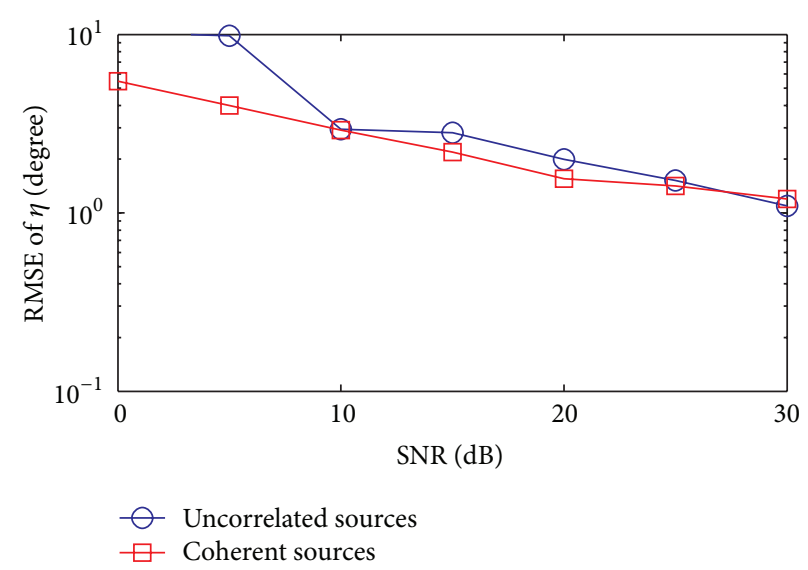

(e)

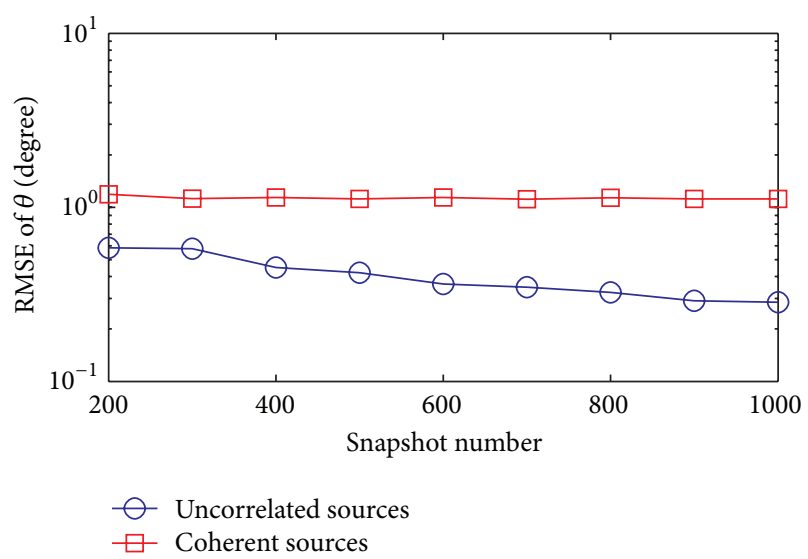

(b)

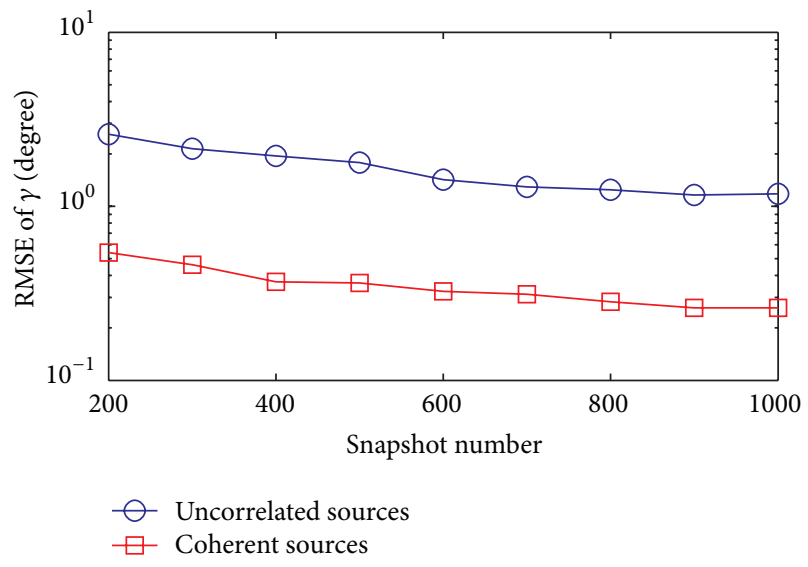

(d)

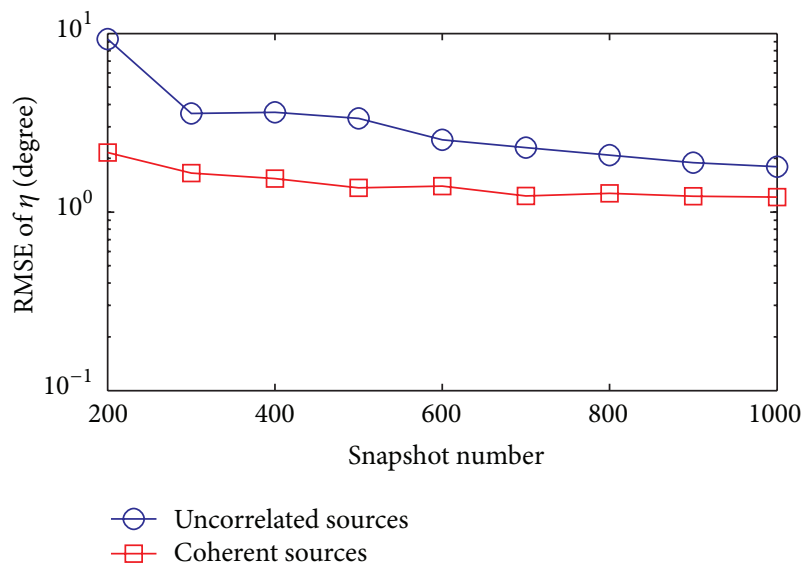

(f)

FIGURE 5: RMSE of angle and polarization parameter estimation versus SNR and snapshot number: (a) RMSE of $\theta$ versus SNR with the fixed snapshot number 500; (b) RMSE of $\theta$ versus snapshot number with the fixed SNR $20 \mathrm{~dB}$; (c) RMSE of $\gamma$ versus SNR with the fixed snapshot number 500; (d) RMSE of $\gamma$ versus snapshot number with the fixed SNR $20 \mathrm{~dB}$; (e) RMSE of $\eta$ versus SNR with the fixed snapshot number 500; (f) RMSE of $\eta$ versus snapshot number with the fixed SNR $20 \mathrm{~dB}$.

sensors is used for the comparative study. The antenna hardware cost of the proposed method is less than that of the comparative methods. One uncorrelated source is parameterized by $\left\{70.1^{\circ}, 15^{\circ},-20^{\circ}\right\}$, and the two coherent sources are parameterized by $\left\{20.7^{\circ}, 30^{\circ}, 16^{\circ}\right\}$ and $\left\{41.6^{\circ}, 13^{\circ}, 50^{\circ}\right\}$ with the fading coefficients $[1,-0.3358-0.7261 j]$. Due to the fact that the PAS and the IPAS methods fail to provide an estimation of polarization parameters, only the RMSE of the angle estimates are considered in this simulation. It can be observed from Figure 6 that the proposed and the IPAS 

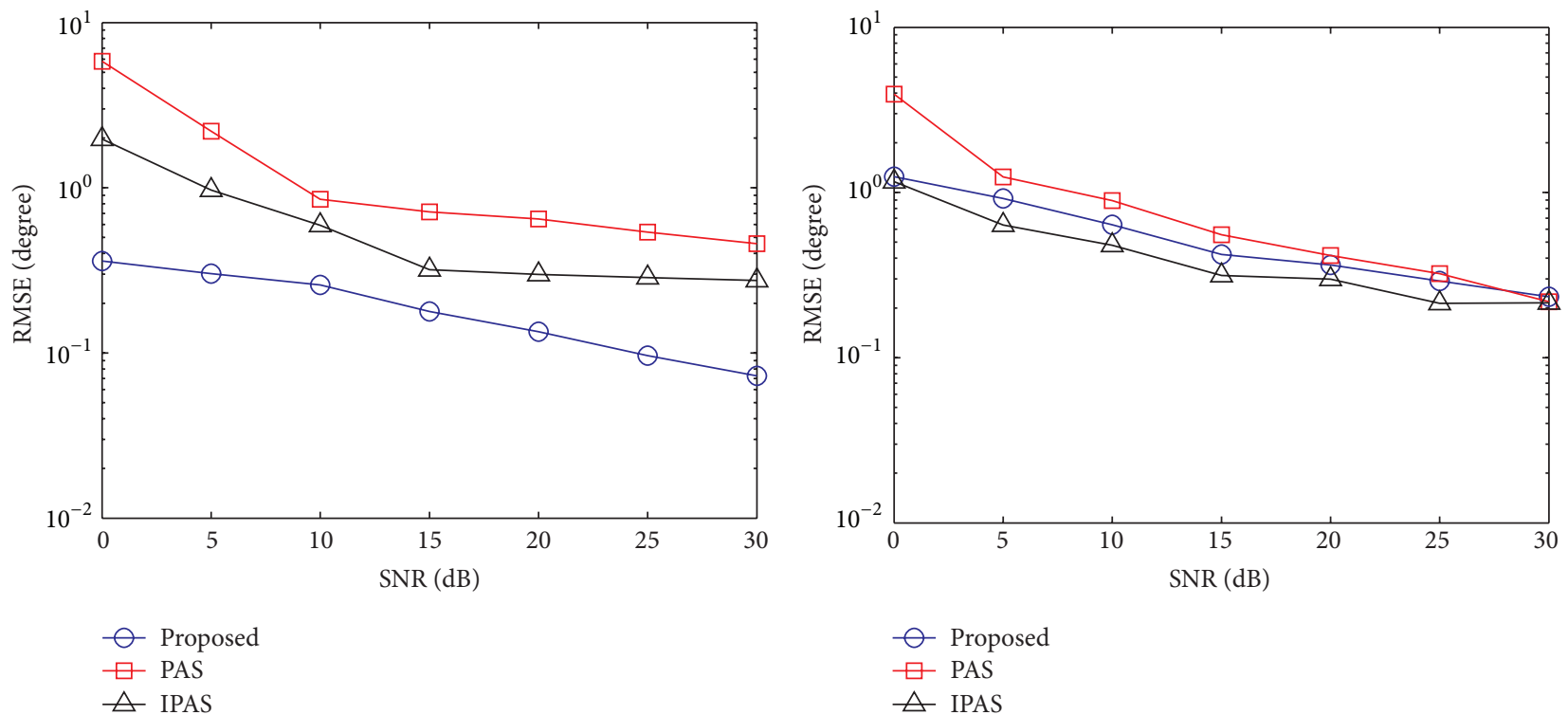

(a)

(b)

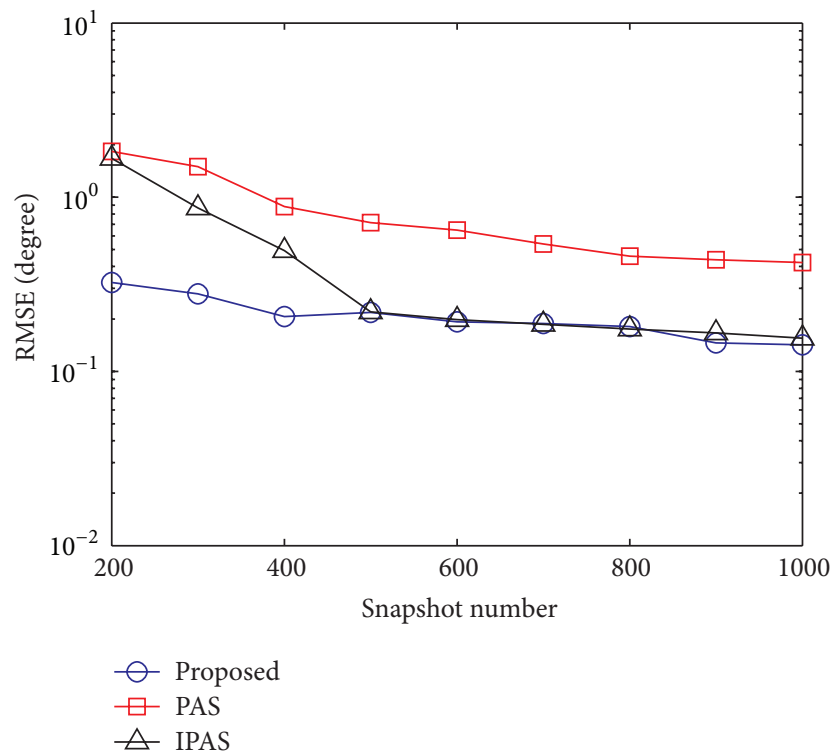

(c)

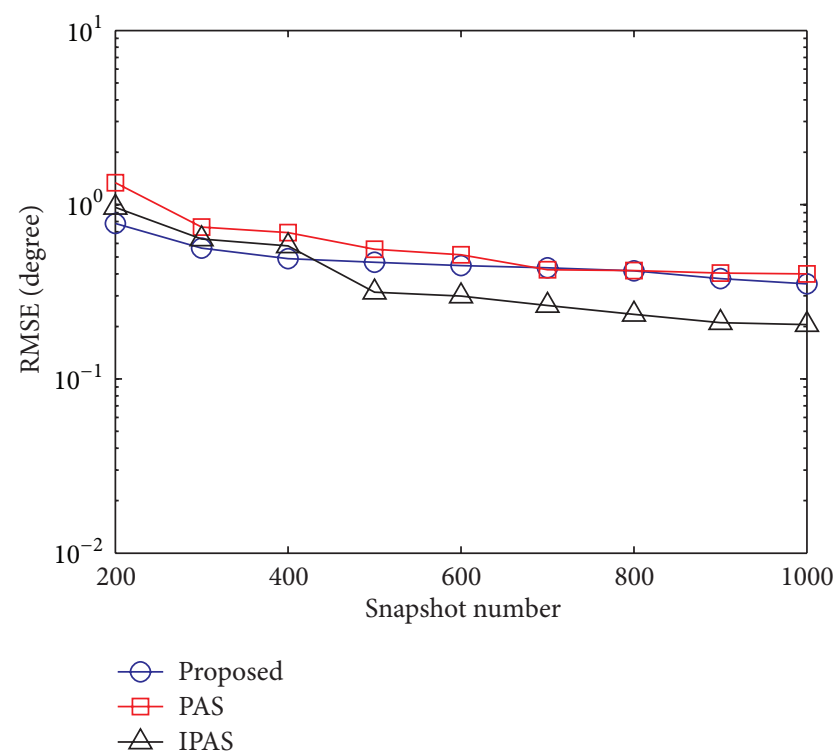

(d)

FIGURE 6: Comparison of RMSE of angle estimation of the proposed method, PAS method, and IPAS method versus SNR and snapshot number: (a) RMSE of uncorrelated angle estimation versus SNR with the fixed snapshot number 500; (b) RMSE of coherent angle estimation versus SNR with the fixed snapshot number 500; (c) RMSE of uncorrelated angle estimation versus snapshot number with the fixed SNR $20 \mathrm{~dB}$; (d) RMSE of coherent angle estimation versus snapshot number with the fixed SNR $20 \mathrm{~dB}$.

methods outperform the PAS method significantly for both the uncorrelated and the coherent sources. Moreover, the performance of the proposed method is superior for the uncorrelated angle estimation and slightly inferior for the coherent angle estimation as compared to the IPAS method. The reason for this superiority is that both the proposed method and the IPAS method estimate the uncorrelated and coherent sources separately. This allows full utilization of the effective array aperture and improves the estimation accuracy accordingly, while the PAS method estimates the uncorrelated and the coherent sources simultaneously with the whole arrays. In addition, the IPAS method estimates the coherent sources by taking an average of the received data from the 6-component vector sensor array, thus acquiring a high accuracy of coherent angle estimations at the expense of the increasing antenna hardware costs in comparison with the dual-polarization vector sensor.

The fourth simulation compares the estimation performance of the proposed method, the PS-MUSIC method, and the PDS-MUSIC method. The arrays used in this simulation are the same as that in the third simulation. Since the PSMUSIC and the PDS-MUSIC methods cannot address the 


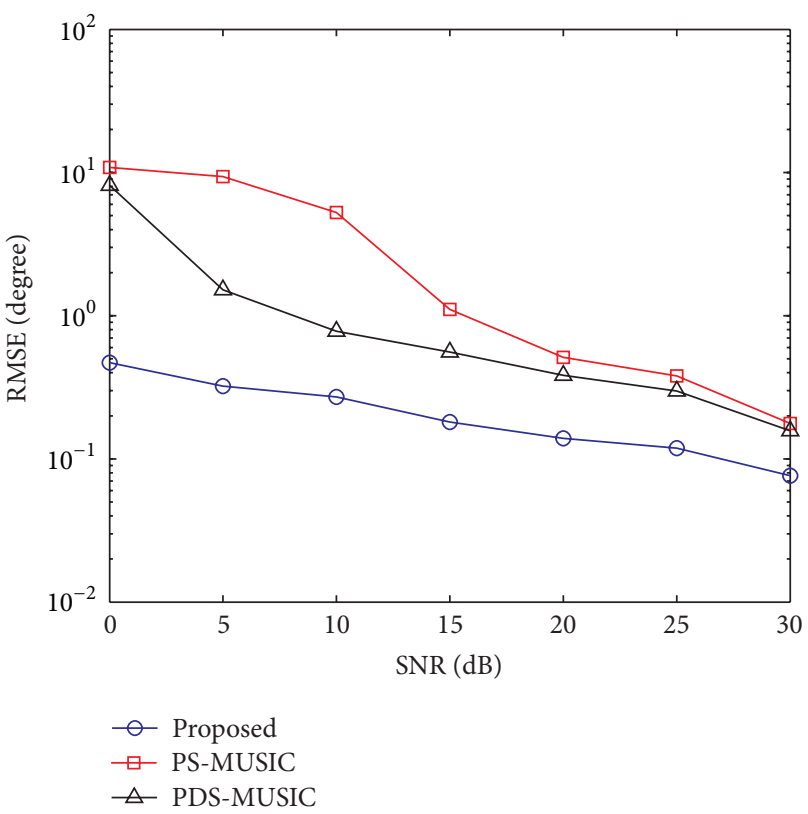

(a)

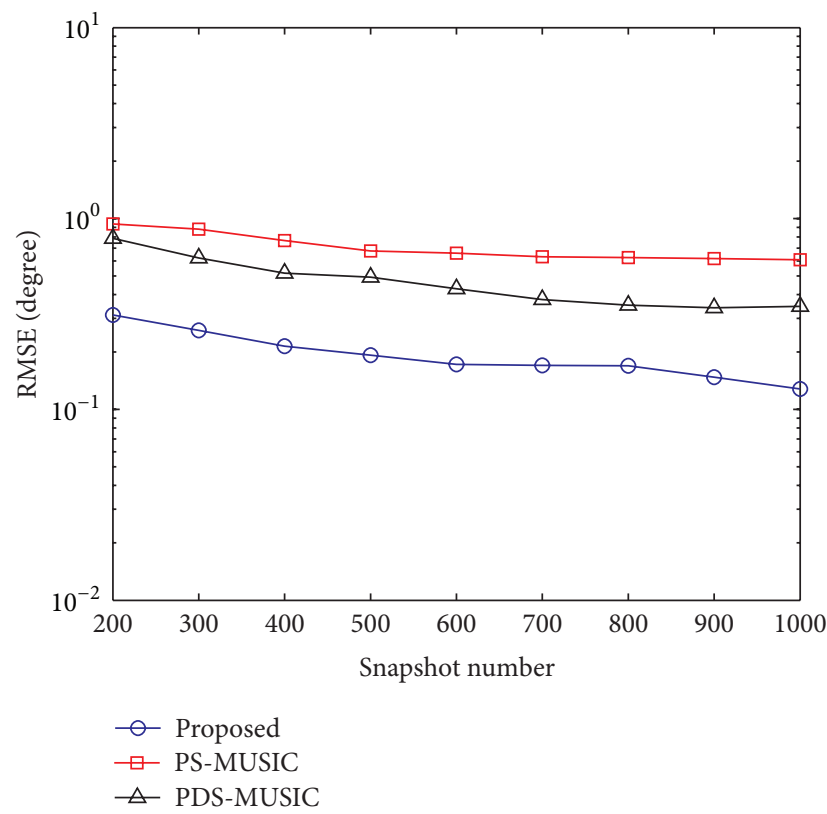

(b)

FIGURE 7: Comparison of RMSE of angle estimation of the proposed, PS-MUSIC, and PDS-MUSIC methods versus SNR and snapshot number: (a) RMSE versus SNR with the fixed snapshot number 500; (b) RMSE versus snapshot number with the fixed SNR $20 \mathrm{~dB}$.

coexistence of the uncorrelated and coherent sources, only the RMSE of coherent angle estimates are considered in this simulation. Two coherent sources are parameterized by $\left\{15.7^{\circ}, 30^{\circ}, 16^{\circ}\right\}$ and $\left\{65.1^{\circ}, 13^{\circ}, 30^{\circ}\right\}$ with the fading coefficients $[1,0.4956+0.4944 j]$ considered in this simulation. It can be seen from Figure 7 that the proposed method outperforms PS-MUSIC and the PDS-MUSIC methods, especially when the SNR is low or the snapshot number is small. The reason is that two rotational-invariant submatrix pairs are extracted from two Hankel matrices, which can provide accurate coherent angle estimates. Restricted by the array model and the method itself, the PDS-MUSIC can estimate at most 3 sources. Moreover, the two comparative methods are coupled with MUSIC method that requires the computationally demanding spectral search.

In the last simulation, we investigate the angular resolution of the proposed method as compared to that of the well-known polarized MUSIC (P-MUSIC) method [9]. The angular resolution is defined as

$$
\begin{aligned}
\Delta \theta_{\min }=\min & (\Delta \theta) \\
\text { s.t. } & \left|\hat{\theta}_{1}-\theta_{1}\right| \leq \frac{\Delta \theta}{2} \\
& \left|\hat{\theta}_{2}-\theta_{2}\right| \leq \frac{\Delta \theta}{2},
\end{aligned}
$$

where $\Delta \theta$ denotes the angular separation between the two incident sources. Consider two uncorrelated sources parameterized by $\left\{57.0^{\circ}, 15^{\circ}, 45^{\circ}\right\}$ and $\left\{57.0^{\circ}+\Delta \theta, 85^{\circ},-68^{\circ}\right\}$ impinging on the 8 -sensor dual-polarization ULA. Figure 8 shows the angular resolution versus SNR and snapshot number, respectively. As observed from Figure 8, the proposed method has a higher angular resolution than the well-known P-MUSIC method. Note that the P-MUSIC requires computationally intensive $3 \mathrm{D}$ spectral search, and the angular resolution of the P-MUSIC method is limited by the search step. This means that if the angular separation between the two sources is less than the search step, these two sources cannot be resolved. In summary, the proposed method offers a higher angular resolution as well as lower computational complexity than the P-MUSIC method.

\section{Conclusion}

In this paper, a computationally efficient angle and polarization estimation method is proposed under the coexistence of uncorrelated and coherent sources by using a dualpolarization vector sensor array. The uncorrelated sources are first distinguished from the coherent sources according to the modulus property of eigenvalues. The uncorrelated angles are then estimated by exploiting the rotational invariance and the associated polarization parameters are obtained from the estimated uncorrelated array response matrix. Finally, two Hankel matrices, constructed from the elements of the estimated coherent array response matrix, are used for the coherent angle estimation, and the corresponding polarization parameters are estimated by solving the least-squares solution to the coherent polarization matrix. In addition, the identifiability and the computational complexity of the proposed method are also discussed. Simulation results show that the proposed method achieves the joint estimation of angle and polarization with a high accuracy as compared with the PS, PDS, PAS, and IPAS methods. Further, the proposed method is found to be computationally efficient and has a higher angular resolution. In the future, research will be 


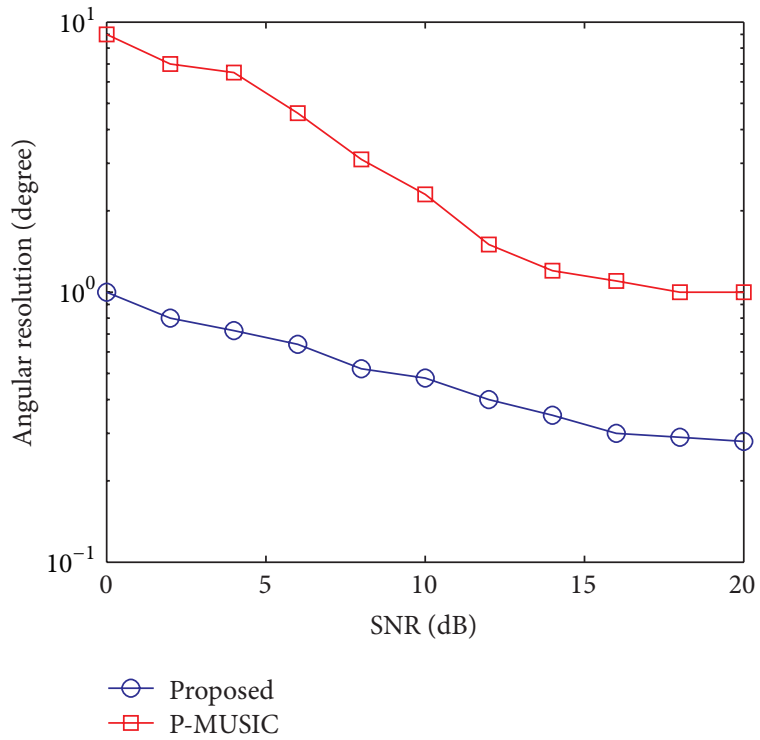

(a)

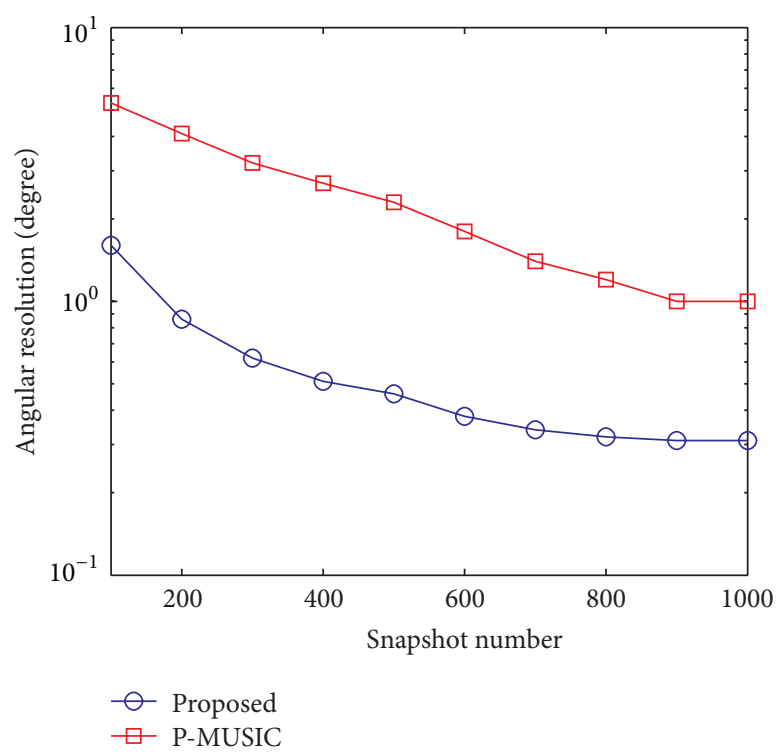

(b)

FIGURE 8: Angular resolution versus SNR and snapshot number: (a) RMSE versus SNR with the fixed snapshot number, 500; (b) RMSE versus snapshot number with the fixed SNR $10 \mathrm{~dB}$.

extended to the joint 2D angle and polarization estimation with the interelement spacing beyond a half-wavelength.

\section{Appendix}

In this appendix, we prove that (32) holds true:

$$
\begin{aligned}
& \mathbf{Q}_{k}=\left(\left[\begin{array}{c}
\mathbf{C}_{c_{k, 1}} \\
\mathbf{C}_{c_{k, 1}} \Delta_{c, k} \\
\vdots \\
\mathbf{C}_{c_{k, 1}} \Delta_{c, k}^{M-p_{k}-1}
\end{array}\right]\left[\begin{array}{llll}
\boldsymbol{c}_{k} & \Delta_{c, k} \boldsymbol{\varsigma}_{k} & \cdots & \Delta_{c, k}^{p_{k}} \boldsymbol{\varsigma}_{k}
\end{array}\right]\right)^{\dagger} \\
& \left(\left[\begin{array}{c}
\mathbf{C}_{c_{k, 2}} \\
\mathbf{C}_{c_{k, 2}} \Delta_{c, k} \\
\vdots \\
\mathbf{C}_{c_{k, 2}} \Delta_{c, k}^{M-p_{k}-1}
\end{array}\right]\left[\begin{array}{llll}
\boldsymbol{c}_{k} & \Delta_{c, k} \boldsymbol{\varsigma}_{k} & \cdots & \Delta_{c, k}^{p_{k}} \boldsymbol{\varsigma}_{k}
\end{array}\right]\right) \\
& =\left(\left(\left[\begin{array}{c}
\mathbf{C}_{c_{k, 1}} \\
\mathbf{C}_{c_{k, 1}} \Delta_{c, k} \\
\vdots \\
\mathbf{C}_{c_{k, 1}} \Delta_{c, k}^{M-p_{k}-1}
\end{array}\right]\left[\begin{array}{llll}
\boldsymbol{c}_{k} & \Delta_{c, k} \boldsymbol{\varsigma}_{k} & \cdots & \Delta_{c, k}^{p_{k}} \boldsymbol{s}_{k}
\end{array}\right]\right)^{H}\right. \\
& \left.\cdot\left(\left[\begin{array}{c}
\mathbf{C}_{c_{k, 1}} \\
\mathbf{C}_{c_{k, 1}} \Delta_{c, k} \\
\vdots \\
\mathbf{C}_{c_{k, 1}} \Delta_{c, k}^{M-p_{k}-1}
\end{array}\right]\left[\begin{array}{llll}
\boldsymbol{c}_{k} & \Delta_{c, k} \boldsymbol{s}_{k} & \cdots & \Delta_{c, k}^{p_{k}} \boldsymbol{\varsigma}_{k}
\end{array}\right]\right)\right)^{-1}
\end{aligned}
$$

$$
\left(\left[\begin{array}{c}
\mathbf{C}_{c_{k, 1}} \\
\mathbf{C}_{c_{k, 1}} \Delta_{c, k} \\
\vdots \\
\mathbf{C}_{c_{k, 1}} \Delta_{c, k}^{M-p_{k}-1}
\end{array}\right]\left[\begin{array}{llll}
\boldsymbol{s}_{k} & \Delta_{c, k} \boldsymbol{\varsigma}_{k} & \cdots & \Delta_{c, k}^{p_{k}} \boldsymbol{s}_{k}
\end{array}\right]\right)^{H}
$$$$
\cdot\left(\left[\begin{array}{c}
\mathbf{C}_{c_{k, 2}} \\
\mathbf{C}_{c_{k, 2}} \Delta_{c, k} \\
\vdots \\
\mathbf{C}_{c_{k, 2}} \Delta_{c, k}^{M-p_{k}-1}
\end{array}\right]\left[\begin{array}{llll}
\boldsymbol{c}_{k} & \Delta_{c, k} \boldsymbol{\varsigma}_{k} & \cdots & \Delta_{c, k}^{p_{k}} \boldsymbol{s}_{k}
\end{array}\right]\right)
$$$$
=\left[\begin{array}{lllll}
\boldsymbol{c}_{k} & \Delta_{c, k} \boldsymbol{\varsigma}_{k} & \cdots & \Delta_{c, k}^{p_{k}} \boldsymbol{\varsigma}_{k}
\end{array}\right]^{\dagger}\left(\left[\begin{array}{c}
\mathbf{C}_{c_{k, 1}} \\
\mathbf{C}_{c_{k, 1}} \Delta_{c, k} \\
\vdots \\
\mathbf{C}_{c_{k, 1}} \Delta_{c, k}^{M-p_{k}-1}
\end{array}\right]^{H}\right.
$$$$
\left.\left.\cdot\left[\begin{array}{c}
\mathbf{C}_{c_{k, 1}} \\
\mathbf{C}_{c_{k, 1}} \Delta_{c, k} \\
\vdots \\
\mathbf{C}_{c_{k, 1}} \Delta_{c, k}^{M-p_{k}-1}
\end{array}\right]\right)^{-1}\left(\begin{array}{llll}
{\left[\boldsymbol{c}_{k}\right.} & \Delta_{c, k} \boldsymbol{c}_{k} & \cdots & \Delta_{c, k}^{p_{k}} \boldsymbol{s}_{k}
\end{array}\right]^{H}\right)^{\dagger}
$$$$
\cdot\left(\left[\begin{array}{lllll}
{\left[\boldsymbol{\varsigma}_{k}\right.} & \Delta_{c, k} \boldsymbol{\varsigma}_{k} & \cdots & \Delta_{c, k}^{p_{k}} \boldsymbol{\varsigma}_{k}
\end{array}\right]^{H}\right)\left(\left[\begin{array}{c}
\mathbf{C}_{c_{k, 1}} \\
\mathbf{C}_{c_{k, 1}} \Delta_{c, k} \\
\vdots \\
\mathbf{C}_{c_{k, 1}} \Delta_{c, k}^{M-p_{k}-1}
\end{array}\right]^{H}\right.
$$ 


$$
\begin{aligned}
& \left.\cdot\left[\begin{array}{c}
\mathbf{C}_{c_{k, 2}} \\
\mathbf{C}_{c_{k, 2}} \Delta_{c, k} \\
\vdots \\
\mathbf{C}_{c_{k, 2}} \Delta_{c, k}^{M-p_{k}-1}
\end{array}\right]\right)\left[\begin{array}{llll}
\boldsymbol{\varsigma}_{k} & \Delta_{c, k} \boldsymbol{\varsigma}_{k} & \cdots & \Delta_{c, k}^{p_{k}} \boldsymbol{\varsigma}_{k}
\end{array}\right] \\
& =\left[\begin{array}{lllll}
\boldsymbol{\varsigma}_{k} & \Delta_{c, k} \boldsymbol{\varsigma}_{k} & \cdots & \Delta_{c, k}^{p_{k}} \boldsymbol{\varsigma}_{k}
\end{array}\right]^{\dagger}\left(\left[\begin{array}{c}
\mathbf{C}_{c_{k, 1}} \\
\mathbf{C}_{c_{k, 1}} \Delta_{c, k} \\
\vdots \\
\mathbf{C}_{c_{k, 1}} \Delta_{c, k}^{M-p_{k}-1}
\end{array}\right]^{\dagger}\right.
\end{aligned}
$$

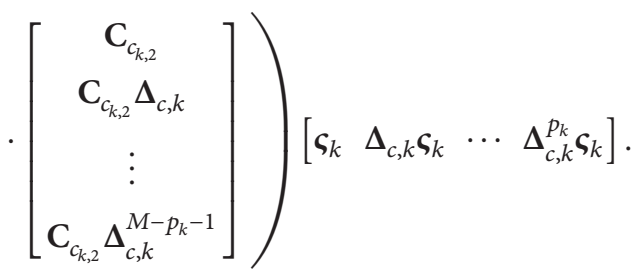

Note that

$$
\left[\begin{array}{c}
\mathbf{C}_{c_{k, 1}} \\
\mathbf{C}_{c_{k, 1}} \Delta_{c, k} \\
\vdots \\
\mathbf{C}_{c_{k, 1}} \Delta_{c, k}^{M-p_{k}-1}
\end{array}\right]^{\dagger}\left[\begin{array}{c}
\mathbf{C}_{c_{k, 2}} \\
\mathbf{C}_{c_{k, 2}} \Delta_{c, k} \\
\vdots \\
\mathbf{C}_{c_{k, 2}} \Delta_{c, k}^{M-p_{k}-1}
\end{array}\right]
$$

can be simplified to a $p_{k} \times p_{k}$ diagonal matrix as

$$
\begin{aligned}
& {\left[\begin{array}{c}
\mathbf{C}_{c_{k, 1}} \\
\mathbf{C}_{c_{k, 1}} \Delta_{c, k} \\
\vdots \\
\mathbf{C}_{c_{k, 1}} \Delta_{c, k}^{M-p_{k}-1}
\end{array}\right]^{\dagger}\left[\begin{array}{c}
\mathbf{C}_{c_{k, 2}} \\
\mathbf{C}_{c_{k, 2}} \Delta_{c, k} \\
\vdots \\
\mathbf{C}_{c_{k, 2}} \Delta_{c, k}^{M-p_{k}-1}
\end{array}\right]} \\
& =\left[\begin{array}{llll}
\mu_{K_{u}+k, 1} & & & \\
& \mu_{K_{u}+k, 2} & & \\
& & \ddots & \\
& & & \mu_{K_{u}+k, p_{k}}
\end{array}\right] \text {, }
\end{aligned}
$$

where $\mu_{K_{u}+k, i}=-\tan \gamma_{K_{u}+k, i} \cos \theta_{K_{u}+k, i} e^{j \eta_{K_{u}+k, i}}, i=1,2, \ldots$, $p_{k}$. Combining with (A.1) and (A.3), the equality in (32) holds.

\section{Competing Interests}

The authors declare that there is no conflict of interests regarding the publication of this paper.

\section{Acknowledgments}

This work is supported by the National Aerospace Science Foundation of China (Grant no. 201401P6001) and
Fundamental Research Funds for the Central Universities (HEUCF150804).

\section{References}

[1] X. Yuan, "Estimating the DOA and the polarization of a polynomial-phase signal using a single polarized vector-sensor," IEEE Transactions on Signal Processing, vol. 60, no. 3, pp. 1270 $1282,2012$.

[2] H. Shi, W. Leng, A. Wang, and T. Guo, "DOA estimation for mixed uncorrelated and coherent sources in multipath environment," International Journal of Antennas and Propagation, vol. 2015, Article ID 636545, 8 pages, 2015.

[3] A. Nehorai and E. Paldi, "Vector-sensor array processing for electromagnetic source localization," IEEE Transactions on Signal Processing, vol. 42, no. 2, pp. 376-398, 1994.

[4] J. I. Mars and C. Paulus, "Vector-sensor array processing for polarization parameters and DOA estimation," EURASIP Journal on Advances in Signal Processing, vol. 2010, Article ID 850265, 13 pages, 2010.

[5] M. Hawes and W. Liu, "Design of fixed beamformers based on vector-sensor arrays," International Journal of Antennas and Propagation, vol. 2015, Article ID 181937, 9 pages, 2015.

[6] C.-H. Lin and W.-H. Fang, "Efficient estimation of signal parameters via rotational invariance technique-based algorithm with automatic pairing for two-dimensional angle and polarisation estimation using crossed dipoles," IET Signal Processing, vol. 8, no. 4, pp. 309-319, 2014.

[7] R. O. Schmidt, "Multiple emitter location and signal parameter estimation," IEEE Transactions on Antennas and Propagation, vol. 34, no. 3, pp. 276-280, 1986.

[8] R. Roy and T. Kailath, "ESPRIT-estimation of signal parameters via rotational invariance techniques," IEEE Transactions on Acoustics, Speech and Signal Processing, vol. 37, no. 7, pp. 984995, 1989.

[9] Q. Cheng and Y. Hua, "Performance analysis of the MUSIC and pencil-MUSIC algorithms for diversely polarized array," IEEE Transactions on Signal Processing, vol. 42, no. 11, pp. 3150-3165, 1994.

[10] K. T. Wong and M. D. Zoltowski, "Self-Initiating MUSICBased direction finding and polarization estimation in spatiopolarizational beamspace," IEEE Transactions on Antennas and Propagation, vol. 48, no. 8, pp. 1235-1245, 2000.

[11] M. D. Zoltowski and K. T. Wong, "Closed-form eigenstructurebased direction finding using arbitrary but identical subarrays on a sparse uniform Cartesian array grid," IEEE Transactions on Signal Processing, vol. 48, no. 8, pp. 2205-2210, 2000.

[12] K. T. Wong, L. Li, and M. D. Zoltowski, "Root-MUSIC-based direction-finding and polarization estimation using diversely polarized possibly collocated antennas," IEEE Antennas and Wireless Propagation Letters, vol. 3, no. 1, pp. 129-132, 2004.

[13] J. Li and R. T. Compton Jr., "Angle and polarization estimation using ESPRIT with a polarization sensitive array," IEEE Transactions on Antennas and Propagation, vol. 39, no. 9, pp. 1376-1383, 1991.

[14] J. Li, "Direction and polarization estimation using arrays with small loops and short dipoles," IEEE Transactions on Antennas and Propagation, vol. 41, no. 3, pp. 379-387, 1993.

[15] K. T. Wong and M. D. Zoltowski, "Closed-form directionfinding with arbitrarily spaced electromagnetic vector-sensors at unknown locations," in Proceedings of the IEEE International 
Conference on Acoustics, Speech and Signal Processing (ICASSP '98), pp. 1949-1952, May 1998.

[16] M. D. Zoltowski and K. T. Wong, "ESPRIT-based 2-D direction finding with a sparse uniform array of electromagnetic vector sensors," IEEE Transactions on Signal Processing, vol. 48, no. 8, pp. 2195-2204, 2000.

[17] D. Rahamim, J. Tabrikian, and R. Shavit, "Source localization using vector sensor array in a multipath environment," IEEE Transactions on Signal Processing, vol. 52, no. 11, pp. 3096-3103, 2004.

[18] J. He, S. Jiang, J. Wang, and Z. Liu, "Polarization difference smoothing for direction finding of coherent signals," IEEE Transactions on Aerospace and Electronic Systems, vol. 46, no. 1, pp. 469-480, 2010.

[19] C. Gu, J. He, X. Zhu, and Z. Liu, "Efficient 2D DOA estimation of coherent signals in spatially correlated noise using electromagnetic vector sensors," Multidimensional Systems and Signal Processing, vol. 21, no. 3, pp. 239-254, 2010.

[20] Y. Xu and Z. Liu, "Polarimetric angular smoothing algorithm for an electromagnetic vector-sensor array," IET Radar, Sonar and Navigation, vol. 1, no. 3, pp. 230-240, 2007.

[21] M. Diao and C. An, "Direction finding of coexisted independent and coherent signals using electromagnetic vector sensor," Journal of Systems Engineering and Electronics, vol. 23, no. 4, pp. 481-487, 2012.

[22] H. Tao, J. Xin, J. Wang, N. Zheng, and A. Sano, "Twodimensional direction estimation for a mixture of noncoherent and coherent signals," IEEE Transactions on Signal Processing, vol. 63, no. 2, pp. 318-333, 2015.

[23] L. M. Novak, S. D. Halversen, G. J. Owirka et al., "Effects of polarization and resolution on the performance of a SAR automatic target recognition system," The Lincoln Laboratory Journal, vol. 8, no. 1, pp. 49-68, 1995.

[24] T. J. Shan, A. Paulraj, and T. Kailath, "On smoothed rank profile tests in eigenstructure methods for directions-of-arrival estimation," IEEE Transactions on Acoustics, Speech and Signal Processing, vol. 35, no. 10, pp. 1377-1385, 1987.

[25] Y. Zhang, Z. Ye, and C. Liu, "Estimation of fading coefficients in the presence of multipath propagation," IEEE Transactions on Antennas and Propagation, vol. 57, no. 7, pp. 2220-2224, 2009.

[26] L. Gan and X. Luo, "Direction-of-arrival estimation for uncorrelated and coherent signals in the presence of multipath propagation," IET Microwaves, Antennas \& Propagation, vol. 7, no. 9, pp. 746-753, 2013. 


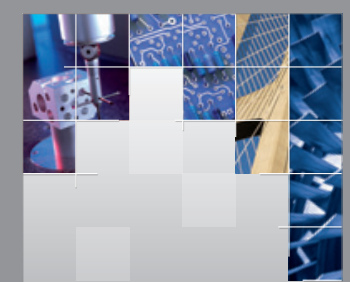

\section{Enfincering}
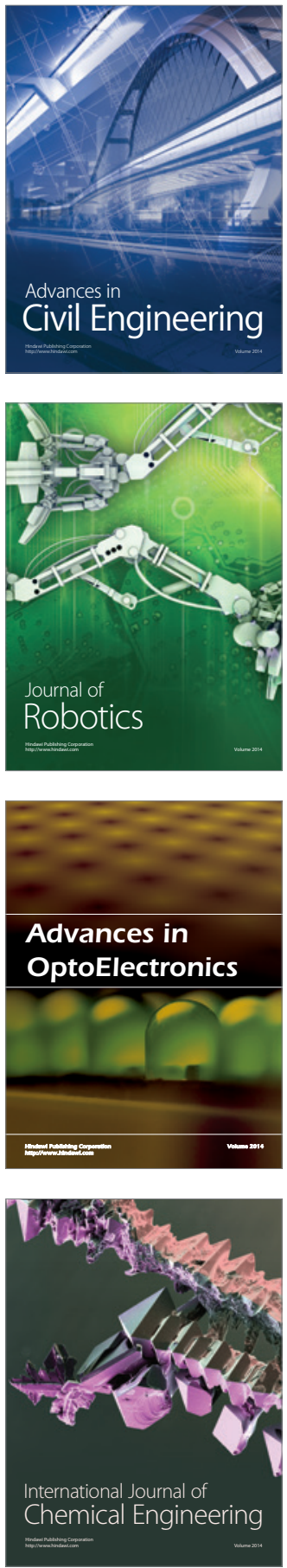

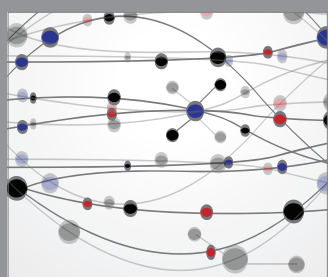

The Scientific World Journal

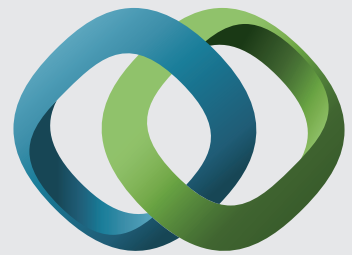

\section{Hindawi}

Submit your manuscripts at

http://www.hindawi.com
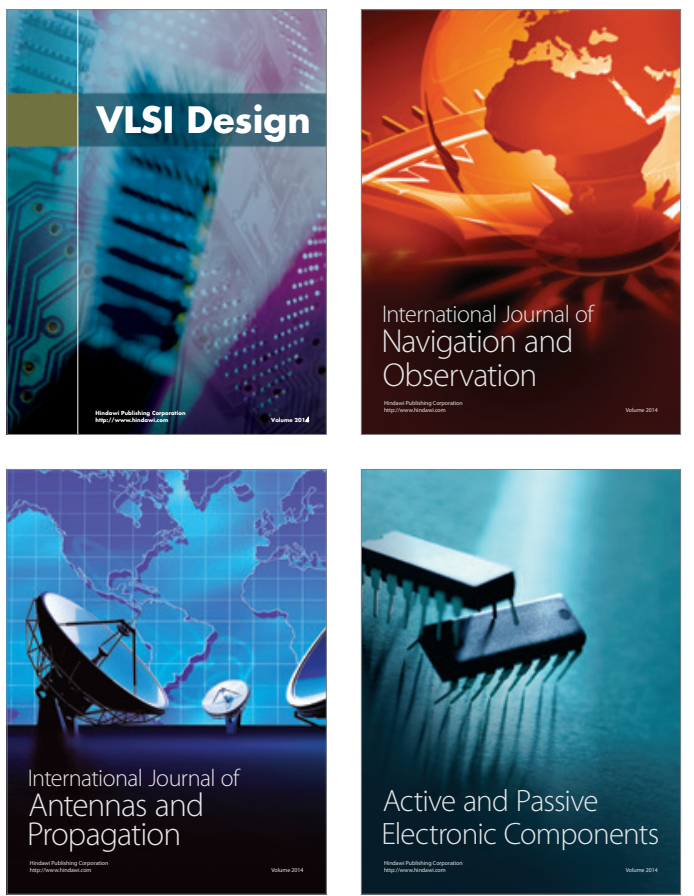
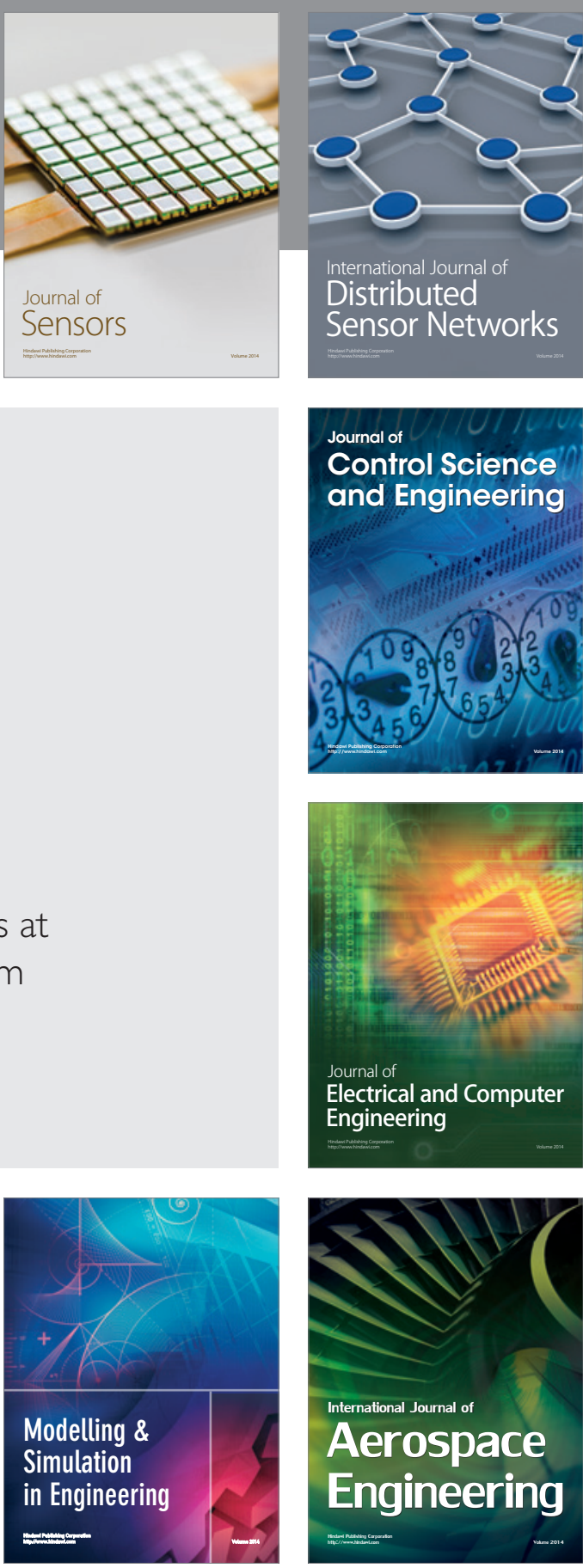

International Journal of

Distributed

Sensor Networks

Journal of

Control Science

and Engineering
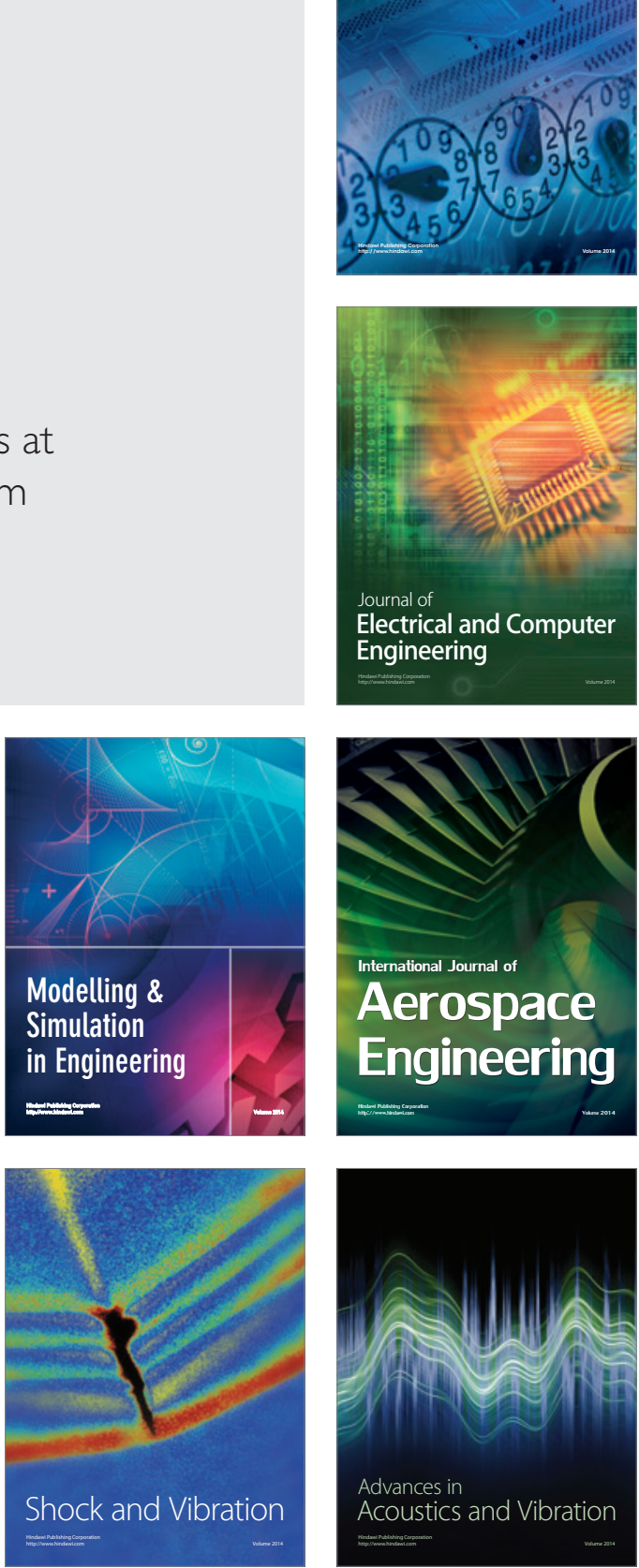